\title{
Vertex operator algebras, minimal models, and modular linear differential equations of order 4
}

\author{
By Yusuke ArIKe, Kiyokazu Nagatomo and Yuichi SAKai \\ Appendix by Don Zagier
}

(Received Apr. 19, 2016)

(Revised Mar. 13, 2017)

\begin{abstract}
In this paper we classify vertex operator algebras with three conditions which arise from Virasoro minimal models: (A) the central charge and conformal weights are rational numbers, (B) the space spanned by characters of all simple modules of a vertex operator algebra coincides with the space of solutions of a modular linear differential equation of order 4 and $(\mathrm{C})$ the dimensions of first three weight subspaces of a VOA are 1,0 and 1, respectively. It is shown that vertex operator algebras which we concern have central charges $c=-46 / 3,-3 / 5,-114 / 7,4 / 5$, and are isomorphic to minimal models for $c=-46 / 3,-3 / 5$ and $\mathbb{Z}_{2}$-graded simple current extensions of minimal models for $c=-114 / 7,4 / 5$.
\end{abstract}

\section{Introduction.}

In the theory of vertex operator algebras (VOAs), one of the most important properties is the modular invariance of the space spanned by characters of all simple modules. This is proved by Zhu [27, Theorem 5.3.3] and Dong, Mason and Li [4, Theorem 1.3] under two conditions- $C_{2}$-cofiniteness and rationality. In the proof of the modular invariance of the space spanned by characters, particularly, convergence of characters, it is shown that there exists a linear differential equation with regular singularity only at $q=0$ whose space of solutions contains the space spanned by characters of all simple modules of a given VOA. This differential equation is now called a monic modular linear differential equation (monic MLDE) and is studied together with the theory of vectorvalued modular forms (cf. [4], [16], [17]). A monic MLDE is defined by means of the Serre derivation $([\mathbf{1 6}],[\mathbf{1 7}])$. The Serre derivation $\vartheta_{k}=q d / d q-(k / 12) E_{2}$ of weight $k$ is a differential operator acting on meromorphic functions on the upper half plane, where $E_{2}$ is the "quasimodular" Eisenstein series. The $i$ th iterated Serre derivation is defined by $\vartheta_{k}^{i}=\vartheta_{k+2(i-1)} \circ \cdots \circ \vartheta_{k+2} \circ \vartheta_{k}$. A linear differential equation $\vartheta_{0}^{p}(f)+\sum_{i=0}^{p-1} P_{i} \vartheta_{0}^{i}(f)=0$ is called a monic MLDE (of weight 0 ) if $P_{i}$ is a holomorphic modular form of weight $2(p-i)$ for each $i$ (see $[\mathbf{1 6}],[\mathbf{1 7}])$.

2010 Mathematics Subject Classification. Primary 81T40; Secondary 11F11, 17B69.

Key Words and Phrases. minimal models, modular invariance, modular linear differential equations, 2D-chiral conformal field theory.

The first author was supported by JSPS KAKENHI Grant Number 25800003. The second author was partially supported by JSPS KAKENHI Grant Number 17K04171, International Center of Theoretical Physics, Italy, and Max Planck institute for Mathematics, Germany. The third author was partially supported by JSPS KAKENHI Grant Numbers 15K13428 and 16H06336. 
One of problems on the study of relationships between VOAs and monic MLDEs is to decide if the space spanned by characters of all simple modules of a VOA coincides with the space of all solutions of a monic MLDE.

One of the most studied examples of VOAs is the simple Virasoro VOA $L\left(c_{p, q}, 0\right)$ (cf. [9], [25]) associated with the irreducible highest weight module of the Virasoro algebra, where the central charge $c_{p, q}$ is $1-6(p-q)^{2} / p q$ for coprime positive integers $p$ and $q$. The VOA $L\left(c_{p, q}, 0\right)$ is often called the minimal model in physics literature. It was shown in [20] and [21] that there is a monic MLDE $L_{p, q}(\vartheta) f=0$ such that the set of characters of simple $L\left(c_{p, q}, 0\right)$-modules forms a fundamental system of solutions of the monic MLDE, where $L_{p, q}(\vartheta)$ is a differential operator of the Serre derivation $\vartheta_{0}$ (We also give a simple proof of this fact in Proposition 6 in Section 3.).

Another example of VOAs is the affine VOA $L_{\mathfrak{g}}(k, 0)$ associated with the irreducible highest weight module of a finite-dimensional simple Lie algebra $\mathfrak{g}$ of positive integral level $k([\mathbf{9}])$. We showed in [1] that if the dimension of the space $\mathcal{X}_{\mathfrak{g}, k}$ spanned by characters of all simple $L_{\mathfrak{g}}(k, 0)$-modules is between 1 and 6 , then $\mathcal{X}_{\mathfrak{g}, k}$ coincides with the space of solutions of a monic MLDE. However, we also found several affine VOAs, $L_{A_{2}}(3,0)$, etc., whose spaces of characters are not the spaces of solutions of any monic MLDEs.

The problem which is intensively studied in this paper is a classification of VOAs whose spaces spanned by characters of all simple modules coincide with the spaces of solutions of monic MLDEs of given orders. Since a monic MLDE of order 1 is $(q d / d q) f=$ 0 , any solution is a constant function. Therefore, the corresponding VOA is trivial. In [19], Mathur, Mukhi and Sen classified the rational conformal field theories whose partition functions (characters) satisfy monic MLDEs of order 2. In the language of VOAs their classification is interpreted as such VOAs are isomorphic to affine VOAs of level 1 associated with (the Deligne exceptional series) $A_{1}, A_{2}, G_{2}, D_{4}, F_{4}, E_{6}, E_{7}$, and the minimal model $L\left(c_{2,5}, 0\right)$. The $3 r d$ order case is studied in [2], [10] and [24]. Since there are infinitely many VOAs whose spaces of characters of simple modules span the spaces of solutions of monic MLDEs of order $3([\mathbf{1}])$, we need an extra condition to obtain finite number of corresponding VOAs. In [2], [10] and [24], it is also assumed that the weight 1 subspace of a VOA is trivial. Then we obtain 8 candidate central charges and show that 6 candidate central charges have corresponding VOAs and the other central charges do not have corresponding $C_{2}$-cofinite and rational VOAs $([\mathbf{3}])$. However, it is not known if each of 6 central charges has the unique corresponding VOA.

Our main purpose of this paper is to classify the VOAs whose spaces spanned by characters of all simple modules coincide with spaces of solutions of monic MLDEs of order 4 under several conditions on VOAs, which may characterize the minimal models.

We first give several results on relations between (extensions of) minimal models and monic MLDEs, which are necessary to prove one of our main theorems. Let $p$ and $q$ be coprime positive integers such that $L\left(c_{p, q}, 0\right)$ does not have positive integral conformal weights. We can prove that if the space of solutions of the monic MLDE $L_{p, q}(\vartheta) f=0$ contains the space spanned by characters of all simple modules of a VOA $V$ with central charge $c_{p, q}$, then $V$ is isomorphic to the minimal model $L\left(c_{p, q}, 0\right)$ (Theorem 8 in Section 3). This result can be considered as a characterization of the minimal models $L\left(c_{p, q}, 0\right)$ for specific pairs $(p, q)$ by means of the monic MLDEs $L_{p, q}(\vartheta) f=0$. 
We also study an extension of a minimal model by its simple module, which is called a $\mathbb{Z}_{2}$-graded simple current extension (SCE). It is known that a $\mathbb{Z}_{2}$-graded SCE of $L\left(c_{p, q}, 0\right)$ uniquely exists if $(p, q)=(2 t+1,4 u+2)$ for some positive integers $t$ and $u$. We also characterize $\mathbb{Z}_{2}$-graded SCEs of minimal models by monic MLDEs. More precisely, we prove that there is a unique monic $\operatorname{MLDE} \hat{L}_{p, q}(\vartheta) f=0$ whose space of solutions coincides with the space spanned by characters of all simple modules of the $\mathbb{Z}_{2}$-graded SCE of $L\left(c_{p, q}, 0\right)$ (Theorem 11 in Section 4 ). Then we show that if the space spanned by characters of all simple modules of a VOA with central charge $c_{p, q}$ is realized by the monic MLDE $\hat{L}_{p, q}(\vartheta) f=0$, then this VOA is isomorphic to the $\mathbb{Z}_{2}$-graded SCE (Theorem 13 in Section 4).

We next prove our main result in this paper. Taking into account of the properties of the minimal models we suppose that a central charge and conformal weights of a VOA $V$ are rational numbers and that $\operatorname{dim} V_{0}=1, \operatorname{dim} V_{1}=0$, and $\operatorname{dim} V_{2}=1$. Then it is shown in [5, Lemma 5.2] that this VOA has no nonzero negative weight spaces, and therefore, the character $\operatorname{ch}_{V}$ of $V$ is expressed as $\operatorname{ch}_{V}=q^{-c / 24}\left(1+q^{2}+m q^{3}+O\left(q^{4}\right)\right)$, where $c$ denotes the central charge of $V$ and $m$ is the dimension of $V_{3}$. By substituting $\operatorname{ch}_{V}$ to the general form of monic MLDEs of order 4, we can determine monic MLDEs uniquely (see Section 5). There are 2 monic MLDEs with generic parameters and 6 monic MLDEs.

The list of monic MLDEs that we obtain contains the monic MLDEs $L_{2,9}(\vartheta) f=0$, $L_{3,5}(\vartheta) f=0, \hat{L}_{5,6}(\vartheta) f=0$ and $\hat{L}_{3,14}(\vartheta) f=0$. It follows from our characterization that VOAs corresponding to these monic MLDEs are isomorphic to the minimal models $L\left(c_{2,9}, 0\right), L\left(c_{3,5}, 0\right)\left(c_{2,9}=-46 / 3, c_{3,5}=-3 / 5\right)$, and the $\mathbb{Z}_{2}$-graded SCEs of minimal models with central charges $c_{5,6}=4 / 5, c_{3,14}=-114 / 7$ by their simple modules with conformal weight 3 , respectively. By solving other monic MLDEs which appear in the paper, we verify that there does not exist VOAs associated with these monic MLDEs and complete a proof of our main result (Theorem 19 in Section 6).

The paper is organized as follows. In Section 1 we review the definitions and the basic properties of vector-valued modular functions, monic MLDEs, and several results obtained by Mason in [17]. The Frobenius method of solving linear differential equations with regular singularities is also explained here. Several important facts and results of the representation theory of the minimal models are illustrated in Section 2. In Section 3 we describe the relations of monic MLDEs and characters of minimal models. The main issue of this section is to give a characterization of minimal models by means of monic MLDEs. In Section 4 we discuss $\mathbb{Z}_{2}$-graded SCEs of minimal models and prove that there is a monic MLDE whose space of solutions coincides with the space spanned by characters of all simple modules of a $\mathbb{Z}_{2}$-graded SCE of a minimal model. A characterization of $\mathbb{Z}_{2}$-graded SCEs of minimal models by means of monic MLDEs and central charges also proved here. In Section 5 we give recursive relations of Fourier coefficients of solutions ( $q$-series) of monic MLDEs of order 4 . We also determine rational numbers which can be central charges and monic MLDEs under our conditions. Finally, we complete a proof of one of our main results in Section 6 by excluding objects which do not fit our conditions. In Appendix, all solutions of the Diophantine equation appeared in Section 5 are determined, which is due to D. Zagier. 


\section{Monic modular linear differential equations and vector-valued mod- ular functions.}

Throughout the paper we denote the complex upper half-plane by $\mathbb{H}=\{\tau \in$ $\mathbb{C} \mid \operatorname{Im} \tau>0\}$. For a positive integer $k \geq 2$, let $E_{2 k}$ be the normalized Eisenstein series of weight $2 k$

$$
E_{2 k}(\tau)=1-\frac{4 k}{B_{2 k}} \sum_{n=1}^{\infty} \sigma_{2 k-1}(n) q^{n}, \quad q=e^{2 \pi \sqrt{-1} \tau} \quad(\tau \in \mathbb{H}),
$$

where $\sigma_{m}(n)$ is the division function and $B_{n}$ is the $n$th Bernoulli number. The quasimodular form $E_{2}(\tau)$ is defined by setting $k=1$ in (1).

DEFINITION. A vector-valued modular function (VVMF) on $S L_{2}(\mathbb{Z})$ is a (column) vector-valued holomorphic function $\mathbb{F}={ }^{t}\left(f_{1}, \ldots, f_{p}\right)$ on $\mathbb{H}$ with a $p$-dimensional complex representation $\rho: S L_{2}(\mathbb{Z}) \rightarrow G L_{p}(\mathbb{C})$ subject to (a) $\mathbb{F}(\gamma(\tau))=\rho(\gamma) \mathbb{F}(\tau)$ for all $\gamma \in S L_{2}(\mathbb{Z})$ and (b) each component function $f_{j}$ has a $q$-expansion $f_{i}=$ $q^{\lambda_{i}} \sum_{n=0}^{\infty} a_{n}^{i} q^{n}\left(a_{0}^{i} \neq 0, \lambda_{i} \in \mathbb{C}\right)$ on $\mathbb{H}$.

A vector-valued modular function $\mathbb{F}$ is called normalized if $f_{i}=q^{\lambda_{i}}(1+$ $\left.\sum_{n=1}^{\infty} a_{n}^{i} q^{n}\right)(1 \leq i \leq m), f_{m+1}, \ldots, f_{p}=0$ and $\lambda_{1}, \ldots, \lambda_{m} f_{j}=0$ are mutually distinct. For a VVMF $\mathbb{F}$, there is an invertible matrix $A$ such that $A \mathbb{F}$ is normalized.

The Serre derivation for $k \in \mathbb{Z}_{\geq 0}$ is defined by

$$
\vartheta_{k}(f)=D(f)-\frac{k}{12} E_{2} f, \quad D=q \frac{d}{d q},
$$

and the $i$ th iterated Serre derivation is defined by $\vartheta_{k}^{i}=\vartheta_{k+2(i-1)} \circ \cdots \circ \vartheta_{k+2} \circ \vartheta_{k}$ with $\vartheta_{k}^{0}=1$. A linear differential equation

$$
\vartheta_{0}^{p}(f)+\sum_{i=0}^{p-1} P_{i} \vartheta_{0}^{i}(f)=0
$$

is called a monic modular linear differential equation (monic MLDE for short) if $P_{i}$ is a holomorphic modular form of weight $2(p-i)$. Since any holomorphic modular form of weight 2 is 0 , we see that $P_{i}=0$ for $i=p-1$. It was shown by Mason in $[\mathbf{1 7}$, Theorem 4.1] that the space of solutions is invariant under the usual slash action of $S L_{2}(\mathbb{Z})$ and that $q=0$ is a unique regular singular point of (2).

TheOREM 1. Let $\mathbb{F}={ }^{t}\left(f_{1}, \ldots, f_{p}\right)$ be a normalized vector-valued modular function. Suppose that $\left\{f_{j}\right\}$ is linearly independent and $f_{i}=q^{\lambda_{i}}\left(1+\sum_{n=1}^{\infty} a_{n}^{i} q^{n}\right)$. Then the set of component functions $\left\{f_{j}\right\}$ forms a fundamental system of a monic modular linear differential equation of order $p$ if and only if $p(p-1)-12 \sum_{i=1}^{p} \lambda_{j}=0$.

REMARK. In this paper we restrict our attention to vector-valued modular functions (vector-valued modular forms of weight 0 ). In [17] all results cited in this section are proved for vector-valued modular forms of non-negative integral weights. 
Since any monic MLDE has a regular singularity at $q=0$, we can use the Frobenius method $([\mathbf{1 1}$, Chapter XVI] $)$ to obtain a fundamental system of solutions of a monic MLDE. Suppose that $f=q^{\rho}\left(1+\sum_{n=0}^{\infty} a_{n} q^{n}\right)$ is a solution of (2). By substituting $f$ to (2) and taking the coefficient of $q^{\rho}$, we see that $\rho$ is a root of the polynomial

$$
\Psi(\rho)=\prod_{k=0}^{p-1}\left(\rho-\frac{k}{6}\right)+\sum_{i=0}^{p-2} P_{i, 0} \prod_{k=0}^{i-1}\left(\rho-\frac{k}{6}\right), \quad P_{i}=\sum_{n=0}^{\infty} P_{i, n} q^{n} .
$$

This polynomial is usually called the indicial polynomial (characteristic polynomial) of the monic MLDE (2) and the roots of the indicial equation, that is, $\Psi(\rho)=0$, are called the indicial roots (indices) of (2).

Lemma 2 ([11, Chapter XVI, p. 397]). If $\lambda$ is an indicial root and there are no indicial roots in $\lambda+\mathbb{Z}_{>0}$, then a solution of the form $q^{\lambda}\left(1+\sum_{n=1}^{\infty} a_{n} q^{n}\right)$ is uniquely determined.

The coefficients $a_{n}$ are determined by recursive relations of Fourier coefficients (see Section 6 for monic MLDEs of order 4).

\section{Virasoro vertex operator algebras.}

In this section we review fundamental properties of Virasoro vertex operator algebras.

The Virasoro algebra denoted here by Vir is a Lie algebra generated by $L_{n}(n \in \mathbb{Z})$ and a central element $C$ with the commutation relations

$$
\left[L_{m}, L_{n}\right]=(m-n) L_{m+n}+\frac{m^{3}-m}{12} \delta_{m,-n} C .
$$

A vertex operator algebra (VOA) is a quadruple $(V, Y, \mathbf{1}, \omega)$ consisting of a $\mathbb{Z}$ graded vector space $V=\bigoplus_{n \in \mathbb{Z}} V_{n}$ with distinguished vectors $\mathbf{1} \in V_{0}$ and $\omega \in V_{2}$, called the vacuum vector and the Virasoro element, respectively, and a linear map $Y: V \rightarrow \operatorname{End}_{\mathbb{C}}(V)\left[\left[z, z^{-1}\right]\right]\left(a \mapsto Y(a, z)=\sum_{n \in \mathbb{Z}} a_{n} z^{-n-1}\right)$ which satisfies a number of axioms (cf. [15], [18]). Define $L_{n}$ for $n \in \mathbb{Z}$ by $Y(\omega, z)=\sum_{n \in \mathbb{Z}} L_{n} z^{-n-2}$. Then the set $\left\{L_{n}, \operatorname{id}_{V} \mid n \in \mathbb{Z}\right\}$ gives a module of the Virasoro algebra on $V$. Particularly, the central element $C$ acts as a scalar $c_{V} \in \mathbb{C}$ and $c_{V}$ is called the central charge of $V$. For each $n \in \mathbb{Z}$, a homogeneous subspace $V_{n}$ is a finite dimensional weight space of weight $n$ with respect to $L_{0}$. A VOA $V$ is said to be of CFT type if $V=\bigoplus_{n=0}^{\infty} V_{n}$ and $V_{0}=\mathbb{C} 1$.

Proposition 3 ([5, Lemma 5.2]). Let $V$ be a vertex operator algebra. If $V_{0}=\mathbb{C} \mathbf{1}$ then $V$ has no nonzero negative weight spaces, that is, $V$ is of CFT type.

Let $V$ be a VOA with central charge $c_{V}$. A weak $V$-module is a pair $\left(M, Y_{M}\right)$ of a vector space $M$ and a linear map $Y_{M}: V \rightarrow \operatorname{End}_{\mathbb{C}}(M)\left[\left[z, z^{-1}\right]\right]$ with naturally required conditions for a module. A weak $V$-module $M$ is also a module of the Virasoro algebra of the central charge $c_{V}$ by letting $Y_{M}(\omega, z)=\sum_{n \in \mathbb{Z}} L_{n} z^{-n-2}$. An admissible $V$-module is a weak $V$-module $M$ which carries a $\mathbb{Z}_{\geq 0}$-grading $M=\bigoplus_{n=0}^{\infty} M(n)$ satisfying $a_{m} M(m) \subseteq$ $M(k+n-m-1)$ for $a \in V_{k}, m, k \in \mathbb{Z}$ and $n \in \mathbb{Z}_{\geq 0}$. A weak $V$-module is said to be a $V$ - 
module if there are finitely many complex numbers $h_{i}$ such that $M=\bigoplus_{i} \bigoplus_{n=0}^{\infty} M_{h_{i}+n}$ where $M_{h}$ is a finite-dimensional eigenspace of $L_{0}$ with eigenvalue $h$. If $M$ is a simple $V$ module, there exists a complex number $h$ such that $M=\bigoplus_{n=0}^{\infty} M_{h+n}$ and $M_{h} \neq 0$. This complex number $h$ is called the conformal weight of a simple $V$-module $M$. The (formal) character of a simple $V$-module $M$ is defined to be

$$
\operatorname{ch}_{M}(\tau)=\operatorname{tr}_{M} q^{L_{0}-c_{V} / 24}=\sum_{n=0}^{\infty}\left(\operatorname{dim} M_{h+n}\right) q^{h-c_{V} / 24+n} .
$$

We can also define the character of a module such as $M=\bigoplus_{n=0}^{\infty} M_{h+n}$ which is not simple. We denote by $\mathcal{X}(V)$ the space spanned by characters of all simple $V$-modules.

It is called that a VOA $V$ is rational if any admissible $V$-module is completely reducible and $C_{2}$-cofinite if the subspace of $V$ spanned by elements of the form $a_{-2} b(a, b \in$ $V$ ) has finite-codimension in $V$. It is shown in [4, Proposition 3.6] that if $V$ is $C_{2}$-cofinite then the number of simple modules is finite up to isomorphisms and each character $\mathrm{ch}_{M}$ of the simple $V$-module $M$ converges on $\mathbb{H}$. Moreover, if $V$ is rational and $C_{2}$-cofinite then the space $\mathcal{X}(V)$ is invariant under the usual slash action of $S L_{2}(\mathbb{Z})$. In other words, the set of characters of all simple $V$-modules forms a finite-dimensional vector-valued modular function.

One of the most important examples of rational and $C_{2}$-cofinite VOAs is the concept of the minimal models. Let $\operatorname{Vir}_{\geq 0}=\bigoplus_{n=0}^{\infty} \mathbb{C} L_{n}$, and $c, h$ be complex numbers. Let $\mathbb{C} v_{c, h}$ be a 1-dimensional $U\left(\operatorname{Vir}_{\geq 0}\right)$-module which is defined by $L_{n} v_{c, h}=h \delta_{n, 0} v_{c, h}$ and $C v_{c, h}=c v_{c, h}$, where $U(\mathfrak{g})$ denotes the universal enveloping algebra of a Lie algebra $\mathfrak{g}$. Then the induced module $M(c, h)=U(\mathrm{Vir}) \otimes_{U\left(\mathrm{Vir}_{>0}\right)} \mathbb{C} v_{c, h}$ is called the Verma module of the Virasoro algebra with central charge $c$ and highest weight $h$, which has the unique maximal proper submodule $J(c, h)$. We denote by $L(c, h)=M(c, h) / J(c, h)$ the irreducible quotient.

Let $\left\langle L_{-1} v_{c, 0}\right\rangle$ be the submodule of $M(c, 0)$, which is generated by $L_{-1} v_{c, 0}$. It is obvious that $\left\langle L_{-1} v_{c, 0}\right\rangle$ is a proper submodule of $M(c, 0)$. We denote the quotient module $M(c, 0) /\left\langle L_{-1} v_{c, 0}\right\rangle$ by $V(c, 0)$ and by 1 the image of $v_{c, 0}$ under the natural surjection. It is shown in $[\mathbf{9}$, Theorem 4.3 and Remark (p. 163)] that $V(c, 0)$ and $L(c, 0)$ are VOAs with the vacuum vectors 1 and the Virasoro elements $\omega=L_{-2} \mathbf{1}$. By Poincaré-BirkhoffWitt theorem applied to $U(\mathrm{Vir})$ the space $V(c, 0)$ has a basis

$$
L_{-n_{1}} \cdots L_{-n_{r}} \mathbf{1} \quad\left(r \geq 0, n_{i} \in \mathbb{Z}, n_{1} \geq \cdots \geq n_{r}>1\right)
$$

whose weight is defined by $L_{0}$, that is, $\sum_{i=1}^{r} n_{i}$. Thus the character of $V(c, 0)$ is given by

$$
q^{-c / 24} \prod_{n>1}\left(1-q^{n}\right)^{-1}=q^{-c / 24}\left(1+q^{2}+q^{3}+2 q^{4}+O\left(q^{5}\right)\right) .
$$

A homogeneous vector $v$ of a highest weight Vir-module is called singular if $L_{n} v=0$ for all positive integer $n$ ( $n=1,2$ are enough by the commutation relations). If $V(c, 0)$ contains a singular vector of positive integral weight, then $V(c, 0)$ is not simple. It was shown in $[\mathbf{6}]$ and $[\mathbf{7}]$ that $V(c, 0)$ contains a singular vector of positive weight if and only if 


$$
c=c_{p, q}=1-\frac{6(p-q)^{2}}{p q}
$$

for coprime positive integers $p$ and $q$. We call the simple Virasoro VOA $L\left(c_{p, q}, 0\right)$ the minimal model. Let $r$ and $s$ be integers, and write

$$
h_{r, s}=\frac{(r q-s p)^{2}-(p-q)^{2}}{4 p q} .
$$

TheOREM 4 ([25, Theorem 4.2]). Let $p, q \in \mathbb{Z}_{>1}$ be coprime integers.

(a) The vertex operator algebra $L\left(c_{p, q}, 0\right)$ is rational and $C_{2}$-cofinite.

(b) For any simple $L\left(c_{p, q}, 0\right)$-module $M$, there exist $1 \leq r \leq p-1$ and $1 \leq s \leq q-1$ such that $M$ is isomorphic to $L\left(c_{p, q}, h_{r, s}\right)$.

(c) Two $L\left(c_{p, q}, 0\right)$-modules $L\left(c_{p, q}, h_{1}\right)$ and $L\left(c_{p, q}, h_{2}\right)$ are isomorphic if and only if $h_{1}=$ $h_{2}$.

(d) As an $L\left(c_{p, q}, 0\right)$-module the rational number $h_{r, s}$ is the conformal weight of $L\left(c_{p, q}, h_{r, s}\right)$.

The number of distinct elements of the set $\left\{h_{r, s} \mid 1 \leq r \leq p-1,1 \leq s \leq q-1\right\}$, which is equal to the number of inequivalent simple $L\left(c_{p, q}, 0\right)$-modules, is $(p-1)(q-1) / 2$ partly since $h_{r, s}=h_{p-r, q-s}$.

Definition. Let $V$ be a vertex operator algebra and $\omega$ its Virasoro element. We denote by $V^{\omega}$ the vertex operator subalgebra of $V$ generated by $\omega$.

In order to study the subalgebra $V^{\omega}$ of a VOA $V$ with central charge $c_{p, q}$, we collect several results from the theory of Verma modules of the Virasoro algebra. It is known ([6], [7] and [12, Theorem 6.5]) that any proper submodule of $M\left(c_{p, q}, 0\right)$ is generated by at most two singular vectors. In particular, the maximal proper submodule $J\left(c_{p, q}, 0\right)$ is generated by $L_{-1} v_{c_{p, q}, 0}$ and a singular vector $w_{p, q}$ of weight $(p-1)(q-1)$. It is also known that any singular vector except $v_{c, 0}$ and $w_{p, q}$ is contained in $\left\langle L_{-1} v_{c_{p, q}, 0}\right\rangle$.

THEOREM 5. Let $V$ be a vertex operator algebra with central charge $c_{p, q}$. Then the vertex operator subalgebra $V^{\omega}$ generated by the Virasoro element $\omega$ of $V$ is isomorphic to either $V\left(c_{p, q}, 0\right)$ or $L\left(c_{p, q}, 0\right)$.

Proof. Because the VOA $V^{\omega}$ is a highest weight Vir-module with central charge $c_{p, q}$, there is a non-zero surjective Vir-homomorphism $\phi: M\left(c_{p, q}, 0\right) \rightarrow V^{\omega}$. Since $L_{-1} \mathbf{1}=0$ in $V$, ker $\phi$ contains $L_{-1} v_{c_{p, q}, 0}$. If $\operatorname{ker} \phi-\left\langle L_{-1} v_{c_{p, q}, 0}\right\rangle$ does not contain a singular vector and is not empty, then $\operatorname{ker} \phi$ cannot be generated by singular vectors, which contradicts to the fact that every non-zero submodule is generated by singular vectors. Therefore, $\operatorname{ker} \phi-\left\langle L_{-1} v_{c_{p, q}, 0}\right\rangle$ contains a singular vector, otherwise it is empty.

If $\operatorname{ker} \phi-\left\langle L_{-1} v_{c_{p, q}, 0}\right\rangle$ contains a singular vector, then it must be $w_{p, q}$. Since the unique maximal submodule is generated by $L_{-1} v_{c_{p, q}, 0}$ and $w_{p, q}$, the submodule $\operatorname{ker} \phi$ is maximal and then $V^{\omega} \cong L\left(c_{p, q}, 0\right)$. 
Suppose that $\operatorname{ker} \phi-\left\langle L_{-1} v_{c_{p, q}, 0}\right\rangle$ is empty. Then $\operatorname{ker} \phi=\left\langle L_{-1} v_{c_{p, q}, 0}\right\rangle$ and $V^{\omega} \cong$ $V\left(c_{p, q}, 0\right)$ follows.

COROLlaRY 1. Let $V$ be a vertex operator algebra with central charge $c_{p, q}$. Suppose that $\operatorname{ch}_{V \omega} \neq \operatorname{ch}_{V\left(c_{p, q}, 0\right)}$. Then $V^{\omega}$ is isomorphic to $L\left(c_{p, q}, 0\right)$.

Proof. Since $\operatorname{ch}_{V^{\omega}} \neq \operatorname{ch}_{V\left(c_{p, q}, 0\right)}$, we have $V^{\omega} \neq V\left(c_{p, q}, 0\right)$. It then follows from Theorem 5 that $V^{\omega}$ is isomorphic to $L\left(c_{p, q}, 0\right)$.

Corollary 2. Let $V$ be a vertex operator algebra. Suppose that $\operatorname{ch}_{V}=\operatorname{ch}_{L\left(c_{p, q}, 0\right)}$. Then $V$ is isomorphic to $L\left(c_{p, q}, 0\right)$.

Proof. By Corollary 1, the VOA $V^{\omega}$ is isomorphic to $L\left(c_{p, q}, 0\right)$ since $\operatorname{ch}_{V^{\omega}} \neq$ $\operatorname{ch}_{V\left(c_{p, q}, 0\right)}$. Therefore $\operatorname{dim} V_{n}=\operatorname{dim} V_{n}^{\omega}$ for each $n$, which proves the corollary since $V^{\omega} \subseteq V$.

\section{Minimal models and monic modular linear differential equations.}

In this section we prove that there exists a monic MLDE whose space of solutions coincides with the space spanned by characters of all simple $L\left(c_{p, q}, 0\right)$-modules. This fact is already known in [21, Theorem 7.1]. Here we give a proof in the context of the theory of VVMFs.

The character $\chi_{r, s}$ of $L\left(c_{p, q}, 0\right)$-module $L\left(c_{p, q}, h_{r, s}\right)$ is given by (cf. [12, Theorem $6.13])$

$$
\begin{aligned}
\chi_{r, s}(q) & =\frac{q^{h_{r, s}-c_{p, q} / 24} \sum_{n \in \mathbb{Z}}\left(q^{r s n^{2}+n(q r-p s)}-q^{r s n^{2}+n(q r+p s)+p q}\right)}{\prod_{n=1}^{\infty}\left(1-q^{n}\right)} \\
& = \begin{cases}q^{-c_{p, q} / 24}\left(1+q^{2}+q^{3}+\cdots\right) & h_{r, s}=0, \\
q^{h_{r, s}-c_{p, q} / 24}\left(1+q+q^{2}+\cdots\right) & \text { otherwise, }\end{cases}
\end{aligned}
$$

which is rewritten as

$$
\chi_{r, s}=\eta(q)^{-1}\left(\theta_{p q, q r-p s}-\theta_{p q, q r+p s}\right),
$$

where $\eta(q)=q^{-1 / 24} \prod_{n=1}^{\infty}\left(1-q^{n}\right)$ is the Dedekind eta function and $\theta_{a, b}=$ $\sum_{n \in \mathbb{Z}} q^{a(n+b / 2 a)^{2}}\left(a, b \in(1 / 2) \mathbb{Z}_{\geq 0}\right)$ is the theta series (see [12, Corollary 6.1]). Since conformal weights of the simple $L\left(c_{p, q}, 0\right)$-modules are mutually distinct, the characters of simple $L\left(c_{p, q}, 0\right)$-modules give an $n=(p-1)(q-1) / 2$-dimensional normalized VVMF.

Proposition 6 ([21, Theorem 7.1]). There exists a unique monic modular linear differential equation $L_{p, q}(\vartheta) f=0$ of order $n=(p-1)(q-1) / 2$ whose space of solutions coincides with the space spanned by characters of all simple $L\left(c_{p, q}, 0\right)$-modules.

ProOF. Since the lowest power in $q$ of the character $\chi_{r, s}$ is $h_{r, s}-c_{p, q} / 24$ by Theorem $4(\mathrm{~d})$, it follows from (3) and (4) that 


$$
\sum_{r=1}^{p-1} \sum_{s=1}^{q-1}\left(h_{r, s}-\frac{c_{p, q}}{24}\right)=\frac{(p-1)(q-1)(p q-p-q-1)}{24} .
$$

Because each term $h_{r, s}$ appears exactly twice in the left-hand side as $h_{r, s}=h_{p-r, q-s}$, we have

$$
\frac{1}{2} \sum_{r=1}^{p-1} \sum_{s=1}^{q-1}\left(h_{r, s}-\frac{c_{p, q}}{24}\right)=\frac{1}{12} \frac{(p-1)(q-1)}{2}\left(\frac{(p-1)(q-1)}{2}-1\right)=\frac{1}{12} n(n-1) .
$$

Hence the proposition follows from Theorem 1.

The central charges and conformal weights of $L\left(c_{p, q}, 0\right)$ with $(p-1)(q-1) / 2<5$ are listed in Table 1 (that is, the number of simple modules is not greater than 4 ). It follows

Table 1. Central charges and conformal weights of $L\left(c_{p, q}, 0\right)$ with $(p-1)(q-1) / 2<5$.

\begin{tabular}{c|c|l}
\hline$(p, q)$ & Central charge & Conformal weights \\
\hline \hline$(2,3)$ & 0 & 0 \\
$(2,5)$ & $-22 / 5$ & $0,-1 / 5$ \\
$(2,7)$ & $-68 / 7$ & $0,-2 / 7,3 / 7$ \\
$(3,4)$ & $1 / 2$ & $0,1 / 2,1 / 16$ \\
$(2,9)$ & $-46 / 3$ & $0,-1 / 3,-5 / 9,-2 / 3$ \\
$(3,5)$ & $-3 / 5$ & $0,-1 / 20,1 / 5,3 / 4$
\end{tabular}

from Theorem 4 and Proposition 6 that the set of indicial roots of $L_{p, q}(\vartheta) f=0$ is $\left\{h_{r, s}-\right.$ $\left.c_{p, q} / 24 \mid 1 \leq r \leq p-1,1 \leq s \leq q-1\right\}$. Since the differential operator $L_{p, q}(\vartheta)$ with $(p-$ 1) $(q-1) / 2<5$ has order less than 5 and any holomorphic modular forms of weight $k<10$ is a scalar multiple of $E_{k}$, we see that $L_{p, q}(\vartheta)=\vartheta_{0}^{n}+\sum_{i=0}^{n-2} \alpha_{i} E_{2(n-i)} \vartheta_{0}^{i}$, where $n=$ $(p-1)(q-1) / 2$. Since a monic MLDE of the form $\vartheta_{0}^{n}(f)+\sum_{i=0}^{n-2} \alpha_{i} E_{2(n-i)} \vartheta_{0}^{i}(f)=0$ is uniquely determined by indicial roots $([\mathbf{1 6}$, Lemma 2.3]), it follows from Table 1 that the differential operators $L_{p, q}(\vartheta)$ with $(p-1)(q-1) / 2<5$ are given by

$$
\begin{aligned}
& L_{2,3}(\vartheta)=\vartheta_{0} \\
& L_{2,5}(\vartheta)=\vartheta_{0}^{2}-\frac{11}{3600} E_{4}, \\
& L_{2,7}(\vartheta)=\vartheta_{0}^{3}-\frac{5}{252} E_{4} \vartheta_{0}+\frac{85}{74088} E_{6}, \\
& L_{3,4}(\vartheta)=\vartheta_{0}^{3}-\frac{107}{2304} E_{4} \vartheta_{0}+\frac{23}{55296} E_{6}, \\
& L_{2,9}(\vartheta)=\vartheta_{0}^{4}-\frac{13}{216} E_{4} \vartheta_{0}^{2}+\frac{53}{5832} E_{6} \vartheta_{0}-\frac{253}{559872} E_{8}, \\
& L_{3,5}(\vartheta)=\vartheta_{0}^{4}-\frac{949}{7200} E_{4} \vartheta_{0}^{2}+\frac{139}{21600} E_{6} \vartheta_{0}-\frac{279}{256000} E_{8}
\end{aligned}
$$

We next give a characterization of a VOA with central charge $c_{p, q}$ whose space spanned by characters of all simple modules is contained in (does not necessarily coincide 
with) the space of solutions of the monic MLDE $L_{p, q}(\vartheta) f=0$. This result will be used to prove Theorem 19 in Section 6. We first show the following proposition.

Proposition 7. Let $V$ be a vertex operator algebra and $M=\bigoplus_{n=0}^{\infty} M_{h+n}$ a $V$ module (not necessarily simple). Suppose that the space $\mathcal{X}(V)$ spanned by characters of all simple $V$-modules is contained in the space of solutions of a monic modular linear differential equation. Then the space of solutions contains the character of $M$.

Proof. Since the number of linearly independent characters of simple $V$-modules is finite and $M_{h+n}$ is finite-dimensional for each $n$, the character of $M$ is a finite sum of characters of simple $V$-modules.

For any pair $(p, q)$ such that the set of conformal weights of simple $L\left(c_{p, q}, 0\right)$-modules does not contain positive integers, we show that the monic $\operatorname{MLDE} L_{p, q}(\vartheta) f=0$ characterizes a VOA with central charge $c_{p, q}$.

THEOREM 8. Let $p$ and $q$ be coprime positive integers such that the set of conformal weights of simple $L\left(c_{p, q}, 0\right)$-modules does not contain positive integers, and let $V$ be a (not necessarily simple) vertex operator algebra with central charge $c_{p, q}$. Suppose that the space of solutions of $L_{p, q}(\vartheta) f=0$ given in Proposition 6 contains the space spanned by characters of all simple $V$-modules. Then $V$ is isomorphic to $L\left(c_{p, q}, 0\right)$.

Proof. It follows from Proposition 7 that the character of $V$ sits in the space of solutions of $L_{p, q}(\vartheta) f=0$. Because $L\left(c_{p, q}, 0\right)$ does not have a positive integral conformal weight and any indicial root is written as $h_{r, s}-c_{p, q} / 24$, the indicial root does not exist in the set $-c_{p, q} / 24+\mathbb{Z}_{>0}$. Since the character $\chi_{1,1}$ of $L\left(c_{p, q}, 0\right)$ is a solution of $L_{p, q}(\vartheta) f=0$, it follows from Lemma 2 that any solution with index $-c_{p, q} / 24$ is a scalar multiple of $\chi_{1,1}$. Therefore, the character of $V$ is $\chi_{1,1}$ since $\operatorname{dim} V_{0}=1$ and the central charge of $V$ is $c_{p, q}$. It follows from Corollary 2 of Theorem 5 that $V$ is isomorphic to $L\left(c_{p, q}, 0\right)$.

If $(p-1)(q-1) / 2<9$, then $L\left(c_{p, q}, 0\right)$ does not have a positive integral conformal weight. However, if $(p, q)=(3,10)$, then $h_{1,9}=2$.

Corollary. Let $p$ and $q$ be coprime positive integers such that $(p-1)(q-1) / 2<$ 9 and $V$ a vertex operator algebra with central charge $c_{p, q}$. If the space spanned by characters of all simple modules is contained in the space of solutions of $L_{p, q}(\vartheta) f=0$, then $V \cong L\left(c_{p, q}, 0\right)$.

\section{Extensions of minimal models and monic modular linear differential equations.}

In this section we discuss the relations between extensions of minimal models and monic MLDEs. We first prove that there exists a monic MLDE $\hat{L}_{p, q}(\vartheta) f=0$ whose space of solutions coincides with the space spanned by characters of all simple modules of the $\mathbb{Z}_{2}$-graded simple current extension of a minimal model with central charge $c_{p, q}$. Our main results in this section is that the monic $\operatorname{MLDE} \hat{L}_{p, q}(\vartheta) f=0$ for the $\mathbb{Z}_{2}$-graded simple current extension determines a VOA with central charge $c_{p, q}$. 
DEFINITION. Let $V$ be a vertex operator algebra and $U$ a vertex operator subalgebra of $V$ which has the same Virasoro element of $V$. Then $V$ is called an extension of $U$. It is called that an extension $V$ of $U$ is called nontrivial if $a_{n} b \neq 0$ for some $a, b \in V \backslash U$ and $n$.

Let $V$ be an extension of a rational VOA $U$. Then $V$ is a finite direct sum of simple $U$ modules with integral conformal weights since $U$ is the $\mathbb{Z}_{\geq 0}$-graded Vir-submodule of $V$.

Let $V$ be a rational and $C_{2}$-cofinite VOA. Suppose that $L, M$ and $N$ are $V$-modules. The fusion rule $N_{M N}^{L}\left(\in \mathbb{Z}_{\geq 0}\right)$ of type $\left(\begin{array}{c}L \\ M N\end{array}\right)$ is the dimension of the space of intertwining operators of type $\left(\begin{array}{c}L \\ M N\end{array}\right)$ (see e.g. [8] for the definition of intertwining operators). The relations among $L, M$ and $N$ are usually denoted by

$$
M \times N=\sum_{L: \text { simple } V \text {-modules }} N_{M N}^{L} L,
$$

and a $V$-module $M$ is called simple current if for any simple $V$-module $N$ there is a simple $V$-module $L$ such that $M \times N=L$.

Definition. Let $V^{0}$ be a vertex operator algebra and $\left\{V^{\alpha} \mid \alpha \in A\right\}$ a set of simple current $V^{0}$-modules indexed by a finite abelian group $A$ such that $V^{\alpha} \times V^{\beta}=V^{\alpha+\beta}$. Then $V=\bigoplus_{\alpha \in A} V^{\alpha}$ is called a $A$-graded simple current extension (SCE) of $V^{0}$ if $(V, Y)$ is a nontrivial extension of $V^{0}$.

Remark. Let $V=\bigoplus_{\alpha \in A} V^{\alpha}$ be a $A$-graded simple current extension and $Y$ the vertex operator on $V$. Then the restriction of the vertex operator

$$
Y: V^{\alpha} \rightarrow \bigoplus_{\gamma \in A} \operatorname{Hom}_{\mathbb{C}}\left(V^{\beta}, V^{\gamma}\right)\left[\left[z, z^{-1}\right]\right]
$$

defines an intertwining operator. Since $V^{\alpha}(\alpha \in A)$ is simple current, that is, $N_{V^{\alpha}, V^{\beta}}^{V^{\gamma}}=$ $\delta_{\alpha+\beta, \gamma}$, we see that $Y\left(v^{\alpha}, z\right) v^{\beta} \in V^{\alpha+\beta}((z))$ for all $v^{\alpha} \in V^{\alpha}$ and $v^{\beta} \in V^{\beta}$. This is one of the reasons why we call this extension $A$-graded.

Theorem 9 (cf. [12, Theorem 9.3]). Let $p, q>1$ be coprime integers. For any integers $0<r_{1}, r_{2}<p$ and $0<s_{1}, s_{2}<q$, we have the fusion rules

$$
L\left(c_{p, q}, h_{r_{1}, s_{1}}\right) \times L\left(c_{p, q}, h_{r_{2}, s_{2}}\right)
$$

Let $\mathcal{A}_{p, q}$ be a free abelian group generated by symbols $\left[L\left(c_{p, q}, h_{r, s}\right)\right]$ labeled by all simple $L\left(c_{p, q}, 0\right)$-modules. Then $\mathcal{A}_{p, q}$ becomes a commutative associative $\mathbb{Z}$-algebra by (6).

It follows from the theorem that

$$
L\left(c_{p, q}, h_{r, s}\right) \times L\left(c_{p, q}, h_{1, q-1}\right)=L\left(c_{p, q}, h_{r, q-s}\right)
$$


which shows that $L\left(c_{p, q}, h_{1, q-1}\right)$ is a simple current module, and that the set $\left\{L\left(c_{p, q}, 0\right), L\left(c_{p, q}, h_{1, q-1}\right)\right\}$ with the fusion product $\times$ forms the abelian group $\mathbb{Z}_{2}$.

Proposition 10 ([26, Lemma 2.6, Theorem 2.14][14, Proposition 3]). Suppose that $h_{1, q-1}$ is a positive integer. Then $V=L\left(c_{p, q}, 0\right) \oplus L\left(c_{p, q}, h_{1, q-1}\right)$ has a unique simple vertex operator algebra structure such that $V$ is a $\mathbb{Z}_{2}$-graded simple current extension of $L\left(c_{p, q}, 0\right)$. Moreover, this extension is rational and $C_{2}$-cofinite.

REMARK. Suppose that $h_{1, q-1}$ is a positive integer. We denote by $\hat{L}\left(c_{p, q}, h_{1, q-1}\right)$ the (unique) $\mathbb{Z}_{2}$-graded simple current extension of $L\left(c_{p, q}, 0\right)$ by $L\left(c_{p, q}, h_{1, q-1}\right)$ given in Proposition 10.

We next prove that there is a monic MLDE whose space of solutions coincides with the space spanned by characters of all simple $\hat{L}\left(c_{p, q}, h_{1, q-1}\right)$-modules. Since $L\left(c_{p, q}, 0\right)$ is rational and $C_{2}$-cofinite, any simple module of the extension $\hat{L}\left(c_{p, q}, h_{1, q-1}\right)$ is a direct sum of simple $L\left(c_{p, q}, 0\right)$-modules. Thus the character of a simple $\hat{L}\left(c_{p, q}, h_{1, q-1}\right)$-module is obtained by the irreducible decomposition of a simple $\hat{L}\left(c_{p, q}, h_{1, q-1}\right)$-module as an $L\left(c_{p, q}, 0\right)$-module.

Suppose that $h_{1, q-1}$ is a positive integer. Since $h_{1, q-1}=(p-2)(q-2) / 4$ and $(p, q)=$ 1 , there exist positive integers $t$ and $u$ such that $(p, q)=(2 t+1,4 u+2)$ or $(4 u+2,2 t+1)$ (we can suppose $(p, q)=(2 t+1,4 u+2)$ since $\left.c_{p, q}=c_{q, p}\right)$. For $1 \leq r \leq p-1$ and $1 \leq s \leq q-1$, we write

$$
\mathbb{Z}_{2}^{r, s}= \begin{cases}\mathbb{Z}_{2} & s=2 u+1 \\ 0 & \text { otherwise }\end{cases}
$$

and $S_{r, s}=\mathbb{Z}_{2} / \mathbb{Z}_{2}^{r, s}$. For every $a \in S_{r, s}$ we introduce the notation

$$
L\left(c_{p, q}, h_{r, s}\right)^{a}= \begin{cases}L\left(c_{p, q}, h_{r, s}\right) & \text { if } a=\overline{0}+\mathbb{Z}_{2}^{r, s}, \\ L\left(c_{p, q}, h_{r, q-s}\right) & \text { if } a=\overline{1}+\mathbb{Z}_{2}^{r, s} .\end{cases}
$$

Then it follows from [26, Theorem 2.14] that a simple $\hat{L}\left(c_{p, q}, h_{1, q-1}\right)$-module, as an $L\left(c_{p, q}, 0\right)$-module, is isomorphic to $\bigoplus_{a \in S_{r, s}} L\left(c_{p, q}, h_{r, s}\right)^{a} \otimes U^{a}$ for some $r$ and $s$, where $U^{a}$ is a simple module of the twisted group algebra $\mathbb{C}^{\lambda_{a}}\left[\mathbb{Z}_{2}^{r, s}\right]$ with 2-cocycle $\lambda_{a} \in$ $Z^{2}\left(\mathbb{Z}_{2}^{r, s}, \mathbb{C}^{\times}\right)$.

The twisted group algebra $\mathbb{C}^{\lambda_{a}}\left[\mathbb{Z}_{2}^{r, s}\right]$ is defined by the group algebra $\mathbb{C}\left[\mathbb{Z}_{2}^{r, s}\right]=$ $\bigoplus_{\alpha \in \mathbb{Z}_{2}^{r, s}} \mathbb{C} e^{\alpha}$ with the deformed multiplication $*$ defined by $e^{\alpha} * e^{\beta}=\lambda_{a}(\alpha, \beta) e^{\alpha+\beta}$ for all $\alpha, \beta \in \mathbb{Z}_{2}^{r, s}$. Since $\mathbb{Z}_{2}^{r, s}$ is either 0 or $\mathbb{Z}_{2}$, each 2-cocycle $\lambda$ satisfies $\lambda_{a}(\alpha, \beta)=\lambda_{a}(\beta, \alpha)$ for all $\alpha, \beta \in \mathbb{Z}_{2}^{r, s}$, which shows that $\mathbb{C}^{\lambda_{a}}\left[\mathbb{Z}_{2}^{r, s}\right]$ is commutative. Thus any simple $\mathbb{C}^{\lambda_{a}}\left[\mathbb{Z}_{2}^{r, s}\right]$ module is 1-dimensional. Therefore, it follows from (7), (8) and $h_{r, s}=h_{p-r, q-s}$ that a simple $\hat{L}\left(c_{p, q}, h_{1, q-1}\right)$-module is isomorphic to one of $L\left(c_{p, q}, 0\right)$-modules

$$
L\left(c_{p, q}, h_{r, s}\right) \oplus L\left(c_{p, q}, h_{r, q-s}\right)(1 \leq r \leq t, 1 \leq s \leq 2 u), \quad L\left(c_{p, q}, h_{r, 2 u+1}\right)(1 \leq r \leq t) .
$$

We remark that these $L\left(c_{p, q}, 0\right)$-modules are not always $\hat{L}\left(c_{p, q}, h_{1, q-1}\right)$-modules.

It follows from [26, Theorem 3.2] that $L\left(c_{p, q}, h_{r, 2 u+1}\right)$ is a simple $\hat{L}\left(c_{p, q}, h_{1, q-1}\right)$ - 
module, and that $L\left(c_{p, q}, h_{r, s}\right) \oplus L\left(c_{p, q}, h_{r, q-s}\right)$ is a simple $\hat{L}\left(c_{p, q}, h_{1, q-1}\right)$-module if $h_{r, s}-$ $h_{r, q-s}$ is an integer. Since $h_{r, s}-h_{r, q-s}=-(2 t+1-2 r)(2 u+1-s) / 2<0$ if $1 \leq s \leq 2 u$, we see that $s$ must be odd $(s=1,3,5, \ldots, 2 u-1)$ and that $h_{r, s}<h_{r, q-s}$ for $1 \leq r \leq t$, $1 \leq s \leq 2 u$. Therefore, every simple $\hat{L}\left(c_{p, q}, h_{1, q-1}\right)$-module as an $L\left(c_{p, q}, 0\right)$-module is isomorphic to one of $L\left(c_{p, q}, h_{r, 2 u+1}\right)$ and $L\left(c_{p, q}, h_{r, 2 v-1}\right) \oplus L\left(c_{p, q}, h_{r, 4 u+3-2 v}\right)$ for $1 \leq r \leq t$ and $1 \leq v \leq u$. This shows that the set of conformal weights of all simple $\hat{L}\left(c_{p, q}, h_{1, q-1}\right)$ modules is given by

$$
\left\{h_{r, 2 u+1} \mid 1 \leq r \leq t\right\} \cup\left\{h_{r, 2 v-1} \mid 1 \leq r \leq t, 1 \leq v \leq u\right\},
$$

and the set of characters of simple $\hat{L}\left(c_{p, q}, h_{1, q-1}\right)$-modules is

$$
\left\{\chi_{r, 2 u+1} \mid 1 \leq r \leq t\right\} \cup\left\{\chi_{r, 2 v-1}+\chi_{r, q-2 v+1} \mid 1 \leq r \leq t, 1 \leq v \leq u\right\} .
$$

THEOREM 11. Let $(p, q)=(2 t+1,4 u+2)$ be a pair of coprime positive integers. Then there is a unique monic modular linear differential equation $\hat{L}_{p, q}(\vartheta) f=0$ of order $t(u+1)$ whose space of solutions coincides with the space spanned by characters of all simple modules of the $\mathbb{Z}_{2}$-graded simple current extension $\hat{L}\left(c_{p, q}, h_{1, q-1}\right)$.

Proof. It follows from (10) with a help of (3) and (4) that

$$
\sum_{r=1}^{t} h_{r, 2 u+1}+\sum_{r=1}^{t} \sum_{v=1}^{u} h_{r, 2 v-1}-\frac{t(u+1) c_{p, q}}{24}=\frac{t(u+1)(t u+t-1)}{12} .
$$

Hence the theorem is proved by Theorem 1 .

REMARK. In [24], it is shown that the character of $\hat{L}\left(c_{p, q}, h_{1, q-1}\right)$ is a solution of a monic MLDE of order $h_{1, q-1}+1$. However, our theorem shows that there is a monic MLDE of order strictly smaller than $h_{1, q-1}+1$. The order of the monic MLDE $\hat{L}_{p, q}(\vartheta) f=$ 0 is $h_{1, q-1}+1$ if and only if one of $p$ and $q$ is 3 and 6 , respectively. For example, the monic MLDE for the extension $\hat{L}\left(c_{5,14}, 9\right)$ obtained in [24] is of order 10, whereas $\hat{L}_{5,14}(\vartheta) f=0$ is of order 8 .

We next show that any VOA $V$ with central charge $c_{p, q}$ whose space spanned by characters of all simple modules is contained in the space of solutions of the monic $\operatorname{MLDE} \hat{L}_{p, q}(\vartheta) f=0$ given in Theorem 11 is isomorphic to $\hat{L}\left(c_{p, q}, h_{1, q-1}\right)$ for specific pairs $(p, q)$ (see Theorem 13 at the end of this section), which will be used to prove Theorem 19 given in Section 6. The main part of a proof of Theorem 13 is to show that $\operatorname{dim} V\left(c_{p, q}, 0\right)_{n}-\operatorname{dim} \hat{L}\left(c_{p, q}, h_{1, q-1}\right)_{n}>0$ for sufficiently large $n$. In order to prove this, we use asymptotic behavior of Fourier coefficients of characters of simple $L\left(c_{p, q}, 0\right)$-modules.

For series $\left\{a_{n}\right\}$ and $\left\{b_{n}\right\}$, we write $a_{n} \sim b_{n}$ if $\lim _{n \rightarrow \infty}\left(a_{n} / b_{n}\right)=1$. It is known in [12, Lemma 9.14] that

$$
\begin{aligned}
\operatorname{dim} L\left(c_{p, q}, h_{r, s}\right)_{h_{r, s}+n} & \\
& \sim \frac{1}{\sqrt{2}} S_{(r, s),\left(r_{0}, s_{0}\right)}\left(\frac{1-6 / p q}{24}\right)^{1 / 4} n^{-3 / 4} e^{\pi \sqrt{(2 / 3)(1-(6 / p q)) n}},
\end{aligned}
$$


where

$$
S_{(r, s),\left(r^{\prime}, s^{\prime}\right)}=(-1)^{(r+s)\left(r^{\prime}+s^{\prime}\right)} \sqrt{\frac{8}{p q}} \sin \left(\frac{\pi r r^{\prime}}{p}(p-q)\right) \sin \left(\frac{\pi s s^{\prime}}{q}(p-q)\right)
$$

and $\left(r_{0}, s_{0}\right)$ is a specific pair of positive integers satisfying $\left|r_{0} q-s_{0} p\right|=1$. Suppose that $(p, q)=(2 t+1,4 u+2)$ for some positive integers $t$ and $u$. It follows that $p-q$ and $s_{0}$ are odd since $\left|2 r_{0}(2 u+1)-2 t s_{0}-s_{0}\right|=1$. Then we have

$$
\begin{aligned}
S_{(1, q-1),\left(r_{0}, s_{0}\right)} & =(-1)^{q\left(r_{0}+s_{0}\right)} \sqrt{\frac{8}{p q}} \sin \left(\frac{\pi r_{0}}{p}(p-q)\right) \sin \left(\frac{\pi(q-1) s_{0}}{q}(p-q)\right) \\
& =(-1)^{q\left(r_{0}+s_{0}\right)} \sqrt{\frac{8}{p q}} \sin \left(\frac{\pi r_{0}}{p}(p-q)\right) \sin \left(\pi s_{0}(p-q)-\frac{\pi s_{0}}{q}(p-q)\right) \\
& =\sqrt{\frac{8}{p q}} \sin \left(\frac{\pi r_{0}}{p}(p-q)\right) \sin \left(\frac{\pi s_{0}}{q}(p-q)\right) \\
& =S_{(1,1),\left(r_{0}, s_{0}\right)}
\end{aligned}
$$

and

$$
\begin{aligned}
S_{(1,1),\left(r_{0}, s_{0}\right)} & =(-1)^{2\left(r_{0}+s_{0}\right)} \sqrt{\frac{8}{p q}} \sin \left(\pi\left(r_{0}-s_{0}\right) \mp \frac{\pi}{p}\right) \sin \left(\pi\left(r_{0}-s_{0}\right) \mp \frac{\pi}{q}\right) \\
& =\sqrt{\frac{8}{p q}} \sin \left(\frac{\pi}{p}\right) \sin \left(\frac{\pi}{q}\right)>0
\end{aligned}
$$

since $\left|r_{0} q-s_{0} p\right|=1$ and $(p, q)=1$. By (12) and (13), the Fourier coefficients of $\chi_{1,1}$ and $\chi_{1, q-1}$ have the same asymptotic behavior at $n \rightarrow \infty$, which implies that

$$
\begin{aligned}
& \operatorname{dim} \hat{L}\left(c_{p, q}, h_{1, q-1}\right)_{n} \\
& \quad \sim \frac{2}{\sqrt{2}} S_{(1,1),\left(r_{0}, s_{0}\right)}\left(\frac{1-6 / p q}{24}\right)^{1 / 4} n^{-3 / 4} e^{\pi \sqrt{(2 / 3)(1-(6 / p q)) n}}>0 .
\end{aligned}
$$

Proposition 12. For sufficiently large $n$, we have $\operatorname{dim} V\left(c_{p, q}, 0\right)_{n}$ $\operatorname{dim} \hat{L}\left(c_{p, q}, h_{1, q-1}\right)_{n}>0$.

Proof. Let $T=U\left(\bigoplus_{n=3}^{\infty} \mathbb{C} L_{-n}\right) v_{c_{p, q}, 0} \subset M\left(c_{p, q}, 0\right)$. It is shown in $[\mathbf{1 2}$, Lemma 9.14] that

$$
\operatorname{dim} T_{n} \sim \frac{\pi^{2}}{12 \sqrt{3} n^{2}} e^{\pi \sqrt{2 n / 3}}
$$

where $T_{n}$ is a graded subspace of $T$ with weight $n$. Since the VOA $V\left(c_{p, q}, 0\right)$ is isomorphic to $S=U\left(\bigoplus_{n=2}^{\infty} \mathbb{C} L_{-n}\right) v_{c_{p, q}, 0}$ as a vector space, we have $\operatorname{dim} V\left(c_{p, q}, 0\right)_{n} \geq \operatorname{dim} T_{n}$ for all $n$. Thus it suffices to prove $\operatorname{dim} T_{n}-\hat{L}\left(c_{p, q}, h_{1, q-1}\right)_{n}>0$ for large enough $n$. Let $A(n)$ and $B(n)$ be the right-hand sides of (14) and (15), respectively. Then it follows from (14) and (15) that there exists a small enough real number $\varepsilon>0$ such that 


$$
\begin{aligned}
& 0<(1-\varepsilon) A(n)<\operatorname{dim} \hat{L}\left(c_{p, q}, h_{1, q-1}\right)_{n}<(1+\varepsilon) A(n), \\
& 0<(1-\varepsilon) B(n)<\operatorname{dim} T_{n}<(1+\varepsilon) B(n)
\end{aligned}
$$

for sufficiently large $n$. Thus we have

$$
\frac{\operatorname{dim} T_{n}}{\operatorname{dim} \hat{L}\left(c_{p, q}, h_{1, q-1}\right)_{n}}>\left(\frac{1-\varepsilon}{1+\varepsilon}\right) \frac{B(n)}{A(n)},
$$

where

$$
\begin{aligned}
\frac{B(n)}{A(n)} & =\frac{\left(\pi^{2} / 12 \sqrt{3} n^{2}\right) e^{\pi \sqrt{2 n / 3}}}{(2 / \sqrt{2}) S_{(1,1),\left(r_{0}, s_{0}\right)}((1-6 / p q) / 24)^{1 / 4} n^{-3 / 4} e^{\pi \sqrt{(2 / 3)(1-(6 / p q) n}}} \\
& =\frac{e^{\pi \sqrt{(2 n / 3)}-\pi \sqrt{(2 / 3)(1-(6 / p q)) n}}\left(\pi^{2} / 12 \sqrt{3}\right)}{n^{5 / 4}(2 / \sqrt{2}) S_{(1,1),\left(r_{0}, s_{0}\right)}((1-6 / p q) / 24)^{1 / 4}} .
\end{aligned}
$$

It follows that $\lim _{n \rightarrow \infty} B(n) / A(n)=\infty$ since $0<1-6 / p q<1$ and $S_{(1,1),\left(r_{0}, s_{0}\right)}>0$. Therefore, $\operatorname{dim} T_{n} / \operatorname{dim} \hat{L}\left(c_{p, q}, h_{1, q-1}\right)_{n} \rightarrow \infty$ as $n \rightarrow \infty$, which proves the proposition.

THEOREM 13. Let $(p, q)=(2 t+1,4 u+2)$ be a pair of coprime positive integers and $V$ a vertex operator algebra with central charge $c_{p, q}$ whose space spanned by characters of all simple $V$-modules is contained in the space of solutions of $\hat{L}_{p, q}(\vartheta) f=0$ given in Theorem 11. Suppose that the set of conformal weights of simple $\hat{L}\left(c_{p, q}, h_{1, q-1}\right)$ does not contain positive integers. Then $V$ is isomorphic to the $\mathbb{Z}_{2}$-graded simple current extension $\hat{L}\left(c_{p, q}, h_{1, q-1}\right)$.

Proof. We first show that $V^{\omega} \cong L\left(c_{p, q}, 0\right)$. Since there are no positive integral conformal weights, it follows from Theorem 11 and Lemma 2 that the character $\chi_{1,1}+\chi_{1, q-1}$ of $\hat{L}\left(c_{p, q}, h_{1, q-1}\right)$ is a unique solution of $\hat{L}_{p, q}(\vartheta) f=0$ of the form $q^{-c_{p, q} / 24}(1+O(q))$. Therefore, the character of $V$ coincides with $\chi_{1,1}+\chi_{1, q-1}$, which shows that $\operatorname{dim} V_{n}=\operatorname{dim} \hat{L}\left(c_{p, q}, h_{1, q-1}\right)_{n}$ for all $n$. It follows from Proposition 12 that $\operatorname{dim} V\left(c_{p, q}, 0\right)_{n}-\operatorname{dim} V_{n}>0$ for sufficiently large $n$. Since $\operatorname{dim} V_{n} \geq \operatorname{dim}\left(V^{\omega}\right)_{n}$, the character of $V^{\omega}$ is not equal to the character of $V\left(c_{p, q}, 0\right)$. Then Corollary 1 of Theorem 5 implies that $V^{\omega}$ is isomorphic to $L\left(c_{p, q}, 0\right)$. Since $L\left(c_{p, q}, 0\right)$ is rational and $C_{2}$-cofinite and the character of $V$ is $\chi_{1,1}+\chi_{1, q-1}$, it follows that $V \cong L\left(c_{p, q}, 0\right) \oplus L\left(c_{p, q}, h_{1, q-1}\right)$ as an $L\left(c_{p, q}, 0\right)$-module.

If $Y(u, z) v=0$ for all $u, v \in L\left(c_{p, q}, h_{1, q-1}\right)$, then the subspace $L\left(c_{p, q}, h_{1, q-1}\right)$ is a $V$-submodule. Since $L\left(c_{p, q}, h_{1, q-1}\right)$ is simple as an $L\left(c_{p, q}, 0\right)$-module, $L\left(c_{p, q}, h_{1, q-1}\right)$ is also a simple $V$-module with positive integral conformal weight $h_{1, q-1}$. Thus $\chi_{1, q-1}$ is a solution of $\hat{L}_{p, q}(\vartheta) f=0$, whereas $\chi_{1, q-1}$ is not a solution of $\hat{L}_{p, q}(\vartheta) f=0$ by (11). This is a contradiction. It then follows from Proposition 10 that $V$ is isomorphic to $\hat{L}\left(c_{p, q}, h_{1, q-1}\right)$.

At the end of the section we give the list of pairs $(p, q)$ such that the differential operator $\hat{L}_{p, q}(\vartheta)$ has order 3 or 4 . By the same argument used to obtain (5) together 
Table 2. Central charges and conformal weights of $\hat{L}\left(c_{p, q}, h_{1, q-1}\right)$ with $h_{1, q-1}<4$.

\begin{tabular}{c|c|c|l}
\hline$(p, q)$ & $h_{1, q}$ & Central charge & Conformal weights \\
\hline \hline$(3,10)$ & 2 & $-44 / 5$ & $0,-1 / 5,-2 / 5$ \\
$(3,14)$ & 3 & $-114 / 7$ & $0,-3 / 7,-4 / 7,-5 / 7$ \\
$(5,6)$ & 3 & $4 / 5$ & $0,1 / 15,2 / 5,2 / 3$
\end{tabular}

with Table 2, the differential operators $\hat{L}_{p, q}(\vartheta)$ for $(p, q)$ in Table 2 are given by

$$
\begin{aligned}
& \hat{L}_{3,10}(\vartheta)=\vartheta_{0}^{3}-\frac{11}{900} E_{4} \vartheta_{0}+\frac{11}{5400} E_{6}, \\
& \hat{L}_{3,14}(\vartheta)=\vartheta_{0}^{4}-\frac{37}{504} E_{4} \vartheta_{0}^{2}+\frac{517}{74088} E_{6} \vartheta_{0}-\frac{57}{87808} E_{8}, \\
& \hat{L}_{5,6}(\vartheta)=\vartheta_{0}^{4}-\frac{67}{900} E_{4} \vartheta_{0}^{2}+\frac{89}{5400} E_{6} \vartheta_{0}-\frac{209}{810000} E_{8} .
\end{aligned}
$$

REMARK. For any pair $(p, q)$ the order of $\hat{L}_{p, q}(\vartheta)$ is strictly greater than 2 .

\section{Monic modular linear differential equations of order 4 and possible central charges.}

In this section we determine candidate central charges of VOAs satisfying the conditions (A) the central charges and conformal weights are rational numbers, (B) the spaces spanned by characters of all simple modules coincide with the spaces of solutions of monic MLDEs of order 4 and (C) $\operatorname{dim} V_{0}=1, \operatorname{dim} V_{1}=0$ and $\operatorname{dim} V_{2}=1$.

REMARKs. (1) Every minimal model $L\left(c_{p, q}, 0\right)$ satisfies all conditions by the definition of $L\left(c_{p, q}, 0\right)$ and Proposition 6.

(2) The condition (C) is the same as the assertion that the first 3 coefficients of the character of a VOA coincide with those of the character $q^{-c / 24} \prod_{n>1}\left(1-q^{n}\right)^{-1}$ of $V(c, 0)$

It is known that a monic MLDE of order 4 can be written as (see [23])

$$
D^{4}(f)-E_{2} D^{3}(f)+\left(3 E_{2}^{\prime}+\alpha_{1} E_{4}\right) D^{2}(f)-\left(E_{2}^{\prime \prime}+\frac{\alpha_{1}}{2} E_{4}^{\prime}-\alpha_{2} E_{6}\right) D(f)+\alpha_{3} E_{8} f=0,
$$

where $D=q(d / d q)$ and $E_{k}^{\prime}=D\left(E_{k}\right), E_{k}^{\prime \prime}=D^{2}\left(E_{k}\right)$. This monic MLDE is in fact rewritten as

$$
\vartheta_{0}^{4}(f)+\left(\alpha_{1}-\frac{11}{36}\right) E_{4} \vartheta_{0}^{2}(f)+\left(\frac{\alpha_{1}}{6}+\alpha_{2}-\frac{5}{216}\right) E_{6} \vartheta_{0}(f)+\alpha_{3} E_{8} f=0,
$$

where we have used the Ramanujan identities:

$$
12 E_{2}^{\prime}=E_{2}^{2}-E_{4}, \quad 3 E_{4}^{\prime}=E_{2} E_{4}-E_{6}, \quad 2 E_{6}^{\prime}=E_{2} E_{4}-E_{8} .
$$


Let $V$ be a (not necessarily simple) VOA with central charge $c$, which satisfies the conditions (A)-(C). By Propositions 3 and 7 , the character $\operatorname{ch}_{V}=$ $q^{-c / 24}\left(1+q^{2}+m q^{3}+O\left(q^{4}\right)\right)$ is a solution of $(17)$, where $m$ is a non-negative integer (the dimension of $V_{3}$ ). By substituting $\mathrm{ch}_{V}$ into (17) and taking the Fourier coefficients of $q^{-c / 24}, q^{1-c / 24}, q^{2-c / 24}$ and $q^{3-c / 24}$, we have

$$
\begin{aligned}
& 576 c^{2} \alpha_{1}-13824 c \alpha_{2}+331776 \alpha_{3}=-c^{4}-24 c^{3}, \\
& 240 c(c+12) \alpha_{1}+12096 c \alpha_{2}+276480 \alpha_{3}=c^{3}+72 c^{2}+576 c, \\
& \left(1244736 c^{2}+29804544 c+1327104\right) \alpha_{1}+(229906944 c+663552) \alpha_{2}+20543901696 \alpha_{3} \\
& \quad=-c^{4}+1896 c^{3}+238464 c^{2}+4257792 c-2654208
\end{aligned}
$$

and

$$
\begin{gathered}
\left(576 c^{2} m+4008960 c^{2}-82944 c m+127733760 c+2985984 m+238878720\right) \alpha_{1} \\
-(13824 c m-1706987520 c-995328 m+334430208) \alpha_{2} \\
+(331776 m+348603678720) \alpha_{3} \\
=-c^{4} m+24 c^{3}(11 m+120)-5184 c^{2}(5 m-88) \\
+41472 c(27 m+296)-1990656(9 m-8) .
\end{gathered}
$$

Since the determinant of the matrix associated with the simultaneous equation (20) is a non-zero scalar multiple of $c(578 c-7)(5 c+22)$, this equation has a unique solution

$$
\begin{aligned}
& \alpha_{1}=-\frac{56 c^{3}+993 c^{2}-11660 c-1440}{96(578 c-7)}, \\
& \alpha_{2}=-\frac{-25 c^{4}-829 c^{3}-7347 c^{2}+1008 c+3456}{1728(578 c-7)}, \\
& \alpha_{3}=-\frac{14 c^{5}+425 c^{4}+3672 c^{3}+5568 c^{2}+9216 c}{110592(578 c-7)}
\end{aligned}
$$

if and only if $c \neq 0,-22 / 5,7 / 578$.

If $c=7 / 578$, then the system (20) is not compatible. Therefore, there does not exist any VOA with central charge $c=7 / 578$ satisfying the conditions (B) and (C). If $c$ is either 0 or $-22 / 5(\operatorname{rank} A=2)$, then the system $(20)$ implies $\left(\alpha_{2}, \alpha_{3}\right)=\left(-2 \alpha_{1}-4,0\right)$ and $\left(17 / 675-\alpha_{1} / 6,1793 / 4320000-11 \alpha_{1} / 3600\right)$, respectively. These cases are studied later in Section 6.

By (21) and (22), the rational number $c$ satisfies

$$
\begin{aligned}
1050 c^{5} & +(5 m+31020) c^{4}+(275600-703 m) c^{3} \\
& +(32992 m+673104) c^{2} a+(504352-517172 m) c+3984 m-210432=0 .
\end{aligned}
$$

We confirmed that this equation does not have rational solutions $c$ for any $3 \leq m \leq 2^{16}$ (according to a private communication with D. Zagier by the 2 nd author, this quintic equation has rational roots even when $m>2^{16}$ ). It is proved by D. Zagier that the rational solutions $(c, m)$ with $m \in \mathbb{Z}_{\geq 0}$ of (23) are given in Table 3 . The proof is given 
in the appendix which is written by him.

Table 3. $m$ and central charges.

\begin{tabular}{c|c}
\hline$m$ & $c$ \\
\hline \hline 1 & $-68 / 7,1 / 2,-3 / 5,-46 / 3$ \\
2 & $-114 / 7,4 / 5$ \\
501971 & 36 \\
3132760 & $122 / 3$ \\
37950512 & $238 / 5$ \\
42987520 & 48
\end{tabular}

For each $c$ given in Table 3 together with $c=0,-22 / 5$, we can obtain indicial equations and indicial roots as in Table 4 (the first column provides candidate conformal weights). Since the sets of indicial roots for $c=36,122 / 3,238 / 5$ and 48 contain nonrational numbers, these cases are excluded by the condition (A). For data in 5th-8th

Table 4. Central charges and indices.

\begin{tabular}{c|c|c}
\hline$c$ & Indices & Indices+c/24 \\
\hline \hline 0 & $0,2,\left(-1 \pm \sqrt{-7-4 \alpha_{1}}\right) / 2$ & $0,2,\left(-1 \pm \sqrt{-7-4 \alpha_{1}}\right) / 2$ \\
$-22 / 5$ & $-1 / 60,11 / 60,\left(25 \pm \sqrt{1114-3600 \alpha_{1}}\right) / 60$ & $-1 / 5,0,\left(14 \pm \sqrt{1114-3600 \alpha_{1}}\right) / 60$ \\
$-68 / 7$ & $-1 / 42,5 / 42,17 / 42,1 / 2$ & $-3 / 7,-2 / 7,0,2 / 21$ \\
$1 / 2$ & $-1 / 48,1 / 24,23 / 48,1 / 2$ & $0,1 / 16,1 / 2,25 / 48$ \\
$-46 / 3$ & $-1 / 36,1 / 12,11 / 36,23 / 36$ & $-2 / 3,-5 / 9,-1 / 3,0$ \\
$-3 / 5$ & $-1 / 40,1 / 40,9 / 40,31 / 40$ & $-1 / 20,0,1 / 5,3 / 4$ \\
$-114 / 7$ & $-1 / 28,3 / 28,1 / 4,19 / 28$ & $-5 / 7,-4 / 7,-3 / 7,0$ \\
$4 / 5$ & $-1 / 30,1 / 30,11 / 30,19 / 30$ & $0,1 / 15,2 / 5,2 / 3$
\end{tabular}

rows of Table 4, we can find corresponding VOAs.

Proposition 14. Let $V$ be a vertex operator algebra with central charge $c$, which satisfies conditions $(\mathrm{A})-(\mathrm{C})$. If $c$ is either $-46 / 3$ or $-3 / 5$, then $V$ is isomorphic to $L(c, 0)$. If $c$ is either $-114 / 7$ or $4 / 5$, then $V$ is isomorphic to $\hat{L}(c, 3)$.

Proof. If the central charge $c$ is either $c=-46 / 3$ or $c=-3 / 5$, Table 4 shows that the corresponding monic MLDE coincides with $L_{2,9}(\vartheta) f=0$ and $L_{3,5}(\vartheta) f=0$ in (5), respectively. Since any conformal weight of simple $L(c, 0)$-module is not a positive integer (see also Table 1), it follows from Theorem 8 that $V$ is isomorphic to $L(c, 0)$.

If the central charge $c$ is either $-114 / 7$ or $4 / 5$, then it follows from Table 2 and Table 4 that the corresponding monic MLDE has order 2 or 3 (see Equation (16)), respectively. Since any conformal weight of simple $\hat{L}(c, 3)$-module is not a positive integer by Table 2 , it follows from Theorem 13 that a VOA $V$ is isomorphic to $\hat{L}(c, 3)$. 


\section{Main results.}

In this section we show that a VOA which satisfies (A) the central charge and conformal weights are rational numbers, (B) there is a monic modular linear differential equation of order 4 whose space of solutions coincides with the space spanned by characters of all simple $V$-modules and (C) $\operatorname{dim} V_{0}=1, \operatorname{dim} V_{1}=0$ and $\operatorname{dim} V_{2}=1$, is isomorphic to one of $L(-46 / 3,0), L(-3 / 5,0), \hat{L}(-114 / 7,3)$ and $\hat{L}(4 / 5,3)$. Since we have already proved in Proposition 14 that central charges $-46 / 3,1 / 2,-114 / 7$ and $4 / 5$ have corresponding VOAs, it suffices to exclude the cases $c=0,-22 / 5,-68 / 7,1 / 2$.

Observe that each central charge in the first 4 rows in Table 4 is equal to $c_{p, q}$ with $(p-1)(q-1) / 2<4$. We prove that there are no VOAs with central charge $c_{p, q}$ satisfying the conditions $(\mathrm{A})-(\mathrm{C})$ for such pairs $(p, q)$.

The indicial equation of the monic MLDE (17) is $\Psi(\rho)=\rho^{4}-\rho^{3}+\alpha_{1} \rho^{2}+\alpha_{2} \rho+\alpha_{3}$ (coefficients $\alpha_{1}, \alpha_{2}$ and $\alpha_{3}$ are uniquely determined by indical roots in Table 4 ). Let $\lambda$ be an indicial root and suppose that other indicial roots do not sit in $\lambda+\mathbb{Z}_{>0}$. Then a solution $q^{\lambda}\left(1+\sum_{n=1}^{\infty} a_{n} q^{n}\right)$ with index $\lambda$ is uniquely determined by the recursive relations

$$
\begin{aligned}
a_{n}=\Psi(\lambda+n)^{-1} & \sum_{m=1}^{n}\left\{e_{2, m}(n-m+\lambda)^{3}-\left(3 m e_{2, m}+\alpha_{1} e_{4, m}\right)(n-m+\lambda)^{2}\right. \\
& \left.+\left(m^{2} e_{2, m}+\frac{1}{2} \alpha_{1} m e_{4, m}-\alpha_{2} e_{6, m}\right)(n-m+\lambda)-\alpha_{3} e_{8, m}\right\} a_{n-m}
\end{aligned}
$$

for each $n \geq 1$.

REMARK. If the indicial equation has multiple roots, then there is a logarithmic solution (see [11, Chapter XVI]). Since characters of simple $V$-modules are power series in $q$, the condition (B) implies that any solution of the monic MLDE (17) does not involve logarithmic terms in $q$. Thus it is enough to consider the case that indicial equations of monic MLDEs of the form (17) do not have multiple roots.

(a) Central charge $c_{2,3}=0$.

The monic MLDE whose set of indicial roots is $\left\{0,2,\left(-1 \pm \sqrt{-7-4 \alpha_{1}}\right) / 2\right\}$ is given by

$$
D^{4}(f)-E_{2} D^{3}(f)+\left(3 E_{2}^{\prime}+\alpha_{1} E_{4}\right) D^{2}(f)-\left(E_{2}^{\prime \prime}+\frac{\alpha_{1}}{2} E_{4}^{\prime}+\left(2 \alpha_{1}+4\right) E_{6}\right) D(f)=0 .
$$

The constant function 1 is a solution with index 0 . It follows from the last remark that the indicial equation does not have multiple roots. We prove that VOAs with central charge 0 , which satisfy the conditions $(\mathrm{A})-(\mathrm{C})$ do not exist.

Let $V$ be a VOA with central charge 0 , which satisfies the conditions $(\mathrm{A})-(\mathrm{C})$. Then the character $\operatorname{ch}_{V}=\sum_{n=0}^{\infty}\left(\operatorname{dim} V_{n}\right) q^{n}=1+q^{2}+m q^{3}+\cdots$ is a solution of (25). Then (21) implies $\alpha_{1}=-2(7 m+664) /(m+912)$. Because all indicial roots are rational numbers, it follows that the indicial roots 


$$
\frac{-1 \pm \sqrt{-7-4 \alpha_{1}}}{2}=-\frac{1}{2} \pm \frac{\sqrt{(49 m-1072)(m+912)}}{m+912}
$$

are rational numbers. Since $m \geq 0$ and the right-hand side of (26) is a rational number, there exists an integer $r$ such that $(49 m-1072)(m+912)=r^{2}$, which is equivalent to $(49 m-7 r+21808)(49 m+7 r+21808)=52349440$. By solving this equation, we have the list of $m$

$\{24,78,88,232,375,708,1194,1285,1636,2608,2733,4236,7908,12400$, $23838,66328,102282,533733\}$.

We can verify by using (26) and (27) on a case-by-case basis that the set of indicial roots does not contain integers greater than 2 . Therefore, the solution $f_{2}$ with index 2 and the first coefficient 1 is uniquely determined by recursive relations (24) as

$$
\begin{aligned}
f_{2}= & q^{2}+m q^{3}+\frac{9\left(853 m^{2}+23392 m-371328\right)}{8(m+2342)} q^{4} \\
& +\frac{3083022 m^{2}+254932344 m^{2}-1529365568 m-46472052736}{(m+2342)(9 m+13928)} q^{5}+O\left(q^{6}\right) .
\end{aligned}
$$

Since 1 is a solution of (25) and $\mathrm{ch}_{V}=1+q^{2}+m q^{3}+O\left(q^{4}\right)$ by the condition (C), we see that $\operatorname{ch}_{V}-1$ is a solution of the monic MLDE with index 2 and the first coefficient 1, which shows that $\operatorname{ch}_{V}=1+f_{2}$. Therefore, all Fourier coefficients of $f_{2}$ must be non-negative integers. Substituting each number of (27) into Fourier coefficients of the solution $f_{2}$, we can show that the Fourier coefficient of $q^{4}$ is an integer if and only if $m \in\{24,232,1636,2608\}$ and that the Fourier coefficient of $q^{5}$ is not an integer ${ }^{1}$ for $m=1636$ and 2608 , and then $m$ is 24 or 232 .

For $m=24$, the set of indicial roots is $\{0,2,-2 / 3,-1 / 3\}$. Since there are no indicial roots in the set $-2 / 3+\mathbb{Z}_{>0}$, the solution $f_{-2 / 3}$ with index $-2 / 3$ is uniquely determined by recursive relations $(24)$ as

$$
f_{-2 / 3}=q^{-2 / 3}\left(1-1448 q-51748 q^{2}+O\left(q^{3}\right)\right) .
$$

Since characters of simple $V$-modules are contained in the space of solutions of (25) (the condition (B)), a scalar multiple of the solution $f_{-2 / 3}$ must coincide with a character of a simple $V$-module. However, this is impossible since the Fourier coefficients of $f_{-2 / 3}$ have alternate signature.

For $m=232$, the corresponding monic MLDE is given by

$$
\vartheta_{0}^{4}(f)-\frac{155}{36} E_{4} \vartheta_{0}^{2}(f)+\frac{715}{216} E_{6} \vartheta_{0}(f)=0
$$

and the set of indicial roots of the monic MLDE is $\{-2,0,1,2\}$.

Let $F_{1}=-\left(E_{10}^{\prime}+264 \eta^{24}\right) / \eta^{22}$ and $F_{2}=\left(E_{4}^{3}-720 \eta^{24}\right)^{\prime} / \eta^{26}$. Then $\eta^{22} F_{1}$ and $\eta^{26} F_{2}$ are extremal quasimodular forms of depth 1 and weight 12 and 14, respectively ([13, Theorem 2.1]). We show that the product $F_{1} F_{2}$ is a solution of the monic MLDE (29).

\footnotetext{
${ }^{1}$ They are $3607786048 / 29$ and $1523763136 / 5$, respectively.
} 
Definition. A quasimodular form of depth $r$ and weight $k$ is a function $f_{0}+f_{1} E_{2}+$ $f_{2} E_{2}^{2}+\cdots+f_{r} E_{2}^{r}$, where $f_{i}$ is a modular form of weight $k-2 i$ and $f_{r} \neq 0$. A quasimodular form of depth $r$ and weight $k$ is called extremal if its $q$-expansion $\sum_{n=0}^{\infty} a_{n} q^{n}$ satisfies

$$
a_{0}=a_{1}=\cdots=a_{m-2}=0, a_{m-1} \neq 0, \quad m=\sum_{i=0}^{r} \operatorname{dim} \mathcal{M}_{k-2 i},
$$

where $\mathcal{M}_{i}$ is the space of modular forms of weight $i$ on $S L_{2}(\mathbb{Z})$.

It is not difficult to verify that

$$
\vartheta_{0}^{2}\left(F_{1}\right)-\frac{143}{144} E_{4} F_{1}=0 \text { and } E_{4} \vartheta_{0}^{2}\left(F_{2}\right)+\frac{1}{3} E_{6} \vartheta_{0}\left(F_{2}\right)-\frac{143}{144} E_{2}^{2} F_{2}=0 .
$$

Since the space of solutions of a (not necessarily monic) MLDE is invariant under the slash action of $S L_{2}(\mathbb{Z})$ (the proof given in [17, p. 385] works), the functions

$$
\begin{aligned}
& F_{1}(-1 / \tau)=\frac{1}{2 \pi \sqrt{-1}}\left(2 \pi \sqrt{-1} \tau F_{1}-E_{10}\right), \\
& F_{2}(-1 / \tau)=\frac{1}{2 \pi \sqrt{-1}}\left(2 \pi \sqrt{-1} \tau F_{2}+E_{4}^{3}-720 \eta^{24}\right),
\end{aligned}
$$

are solutions of (30), respectively. It then follows from (30) that

$$
\begin{aligned}
& \vartheta_{0}\left(F_{1} F_{2}\right)=\vartheta_{0}\left(F_{1}\right) F_{2}+F_{1} \vartheta_{0}\left(F_{2}\right), \\
& \vartheta_{0}^{2}\left(F_{1} F_{2}\right)=4 \vartheta_{0}\left(F_{1}\right) \vartheta_{0}\left(F_{2}\right)-\frac{E_{6}}{3 E_{4}} F_{1} \vartheta_{0}\left(F_{2}\right), \\
& \vartheta_{0}^{4}\left(F_{1} F_{2}\right)=-\frac{715}{216} E_{6} \vartheta_{0}\left(F_{1}\right) F_{2}-\frac{1025}{216} E_{6} F_{1} \vartheta_{0}\left(F_{2}\right)+\frac{155}{9} E_{4} \vartheta_{0}\left(F_{1}\right) \vartheta_{0}\left(F_{2}\right),
\end{aligned}
$$

which shows that $F_{1} F_{2}$ is a solution of the monic MLDE (29). Because the space of solutions of $(29)$ is invariant under the action of $S L_{2}(\mathbb{Z})$, the function

$$
F_{1}(-1 / \tau) F_{2}(-1 / \tau)=\left(\frac{1}{2 \pi \sqrt{-1}}\right)^{2}\left(2 \pi \sqrt{-1} \tau F_{1}-E_{10}\right)\left(2 \pi \sqrt{-1} \tau F_{2}+E_{4}^{3}-720 \eta^{24}\right)
$$

is also a solution of (29) by the modular invariance property. Since the condition (B) implies that the space of solutions of (29) is spanned by power series in $q$, this is a contradiction.

Summarizing these arguments, we have the proposition.

Proposition 15. Vertex operator algebras with central charge $c=0$ satisfying (A)-(C) do not exist.

(b) Central charge $c_{2,5}=-22 / 5$.

The associated monic MLDE (17) is given by 


$$
\begin{aligned}
D^{4}(f)- & E_{2} D^{3}(f)+\left(3 E_{2}^{\prime}+\alpha_{1} E_{4}\right) D^{2}(f) \\
& -\left\{E_{2}^{\prime \prime}+\frac{\alpha_{1}}{2} E_{4}^{\prime}-\left(\frac{17}{675}-\frac{\alpha_{1}}{6}\right) E_{6}\right\} D(f)+\left(\frac{1793}{4320000}-\frac{11 \alpha_{1}}{3600}\right) E_{8} f=0 .
\end{aligned}
$$

The set of indicial roots is obtained as $\left\{-1 / 60,11 / 60,\left(25 \pm \sqrt{1114-3600 \alpha_{1}}\right) / 60\right\}$. Further, it follows by (18) and (19) that (31) is rewritten as

$$
\vartheta_{6} \circ \vartheta_{4} \circ L_{2,5}(\vartheta) f+\left(\alpha_{1}-\frac{121}{400}\right) E_{4} L_{2,5}(\vartheta) f=0 .
$$

Proposition 6 and Table 1 together with the monic MLDE $L_{2,5}(\vartheta) f=0$ in (5) imply that the space of solutions of (32) contains characters $\chi_{1,1}$ and $\chi_{1,2}$ of simple $L(-22 / 5,0)$ modules. Therefore, we see that $\chi_{1,1}$ is a solution with index $11 / 60$ of the monic MLDE (31).

Proposition 16. Vertex operator algebras with central charge $c=-22 / 5$ satisfying the conditions $(\mathrm{A})-(\mathrm{C})$ do not exist.

Proof. Suppose that there is a VOA $V$ with central charge $-22 / 5$, which satisfies the conditions $(\mathrm{A})-(\mathrm{C})$. Then the condition $(\mathrm{B})$ implies that $\mathcal{X}(V)$ coincides with the space of solutions of the monic MLDE (31) whose set of indicial roots is $\left\{-1 / 60,11 / 60,\left(25 \pm \sqrt{1114-3600 \alpha_{1}}\right) / 60\right\}$. If there are no indicial roots in $11 / 60+\mathbb{Z}_{>0}$, then it follows from the condition (C) that the character of $V$ is $\chi_{1,1}$ and that $V \cong L(-22 / 5,0)$ by Corollary 2 of Theorem 5 , which contradicts to the condition (B) since the number of simple $L(-22 / 5,0)$-modules is 2 .

Suppose that there is an indicial root in $11 / 60+\mathbb{Z}_{>0}$. Then there exists a positive integer $\ell$ such that $\left(25+\sqrt{1114-3600 \alpha_{1}}\right) / 60=11 / 60+\ell$. Then the set of indicial roots is

$$
\left\{\frac{13}{20}-\ell,-\frac{1}{60}, \frac{11}{60}, \frac{11}{60}+\ell\right\} .
$$

Since there are no indicial roots in $13 / 20-\ell+\mathbb{Z}_{>0}$, the solution $f_{13 / 20-\ell}$ with in$\operatorname{dex} 13 / 20-\ell$ is written as

$$
f_{13 / 20-\ell}=q^{13 / 20-\ell}\left(1-\frac{6\left(13500 \ell^{4}-20475 \ell^{3}+9525 \ell^{2}-968 \ell-182\right)}{(3 \ell-5)(15 \ell-22)(15 \ell-11)} q+O\left(q^{2}\right)\right)
$$

by the recursive relations (24), and a scalar multiple of $f_{13 / 20-\ell}$ is a character of a simple $V$-module. However, it follows that $\left(13500 \ell^{4}-20475 \ell^{3}+9525 \ell^{2}-968 \ell-182\right) /(3 \ell-$ $5)(15 \ell-22)(15 \ell-11)$ is positive for all $\ell \in \mathbb{Z}_{>0}$, which shows that any scalar multiple of $f_{13 / 20-\ell}$ cannot be a character of a simple $V$-module since the second Fourier coefficient is negative. Thus we have proved the proposition.

\section{(c) Central charge $c_{2,7}=-68 / 7$.}

The associated monic MLDE (17) is written as 


$$
\begin{aligned}
D^{4}(f)-E_{2} D^{3}(f) & +\left(3 E_{2}^{\prime}+\frac{2}{7} E_{4}\right) D^{2}(f) \\
& -\left(E_{2}^{\prime \prime}+\frac{1}{7} E_{4}^{\prime}+\frac{619}{37044} E_{6}\right) D(f)-\frac{85}{148176} E_{8} f=0
\end{aligned}
$$

and the set of indicial roots is $\{-1 / 42,5 / 42,17 / 42,1 / 2\}$. It follows from (5) and (19) that the left-hand side of this monic MLDE is rewritten as

$$
\vartheta_{6}\left(\vartheta_{0}^{3}(f)-\frac{5}{252} E_{4} \vartheta_{0}(f)+\frac{85}{74088} E_{6} f\right)=\vartheta_{6} \circ L_{2,7}(\vartheta) f .
$$

Therefore, the character $\chi_{1,1}$ of $L(-68 / 7,0)$ is a solution of (33) by Table 1 and (5).

Proposition 17. Vertex operator algebras with central charge $c=-68 / 7$ satisfying the conditions $(\mathrm{A})-(\mathrm{C})$ do not exist.

Proof. Suppose that there is a VOA with central charge $-68 / 7$, which satisfies the conditions $(A)-(C)$. Since there are no indicial roots which sit in $17 / 42+\mathbb{Z}_{>0}$, the character of $V$ must be $\chi_{1,1}$. Thus it follows from Corollary 2 of Theorem 5 that $V$ is isomorphic to $L(-68 / 7,0)$ by Lemma 2 . However, the space spanned by characters of all simple $L(-68 / 7,0)$-modules is 3 -dimensional, which contradicts to the condition (B).

(d) Central charge $c_{3,4}=1 / 2$.

The associated monic MLDE is given by

$$
\begin{aligned}
D^{4}(f)-E_{2} D^{3}(f) & +\left(3 E_{2}^{\prime}+\frac{199}{768} E_{4}\right) D^{2}(f) \\
& -\left(E_{2}^{\prime \prime}+\frac{199}{1536} E_{4}^{\prime}+\frac{229}{55296} E_{6}\right) D(f)-\frac{23}{110592} E_{8} f=0 .
\end{aligned}
$$

The set of incidial roots is $\{-1 / 48,23 / 48,1 / 24,1 / 2\}$. It follows from (5) and (19) that the left-hand side of this monic MLDE is rewritten as

$$
\vartheta_{6} \circ\left(\vartheta_{0}^{3}(f)-\frac{107}{2304} E_{4} \vartheta_{0}(f)+\frac{23}{55296} E_{6} f\right)=\vartheta_{6} \circ L_{3,4}(\vartheta) f .
$$

Therefore, the character $\chi_{1,1}$ of $L(1 / 2,0)$ is a solution of the monic MLDE (35) by Table 1 and (5).

Proposition 18. Vertex operator algebras with central charge $1 / 2$ satisfying the conditions (A)-(C) do not exist.

Proof. Suppose that there is a VOA $V$ with central charge $1 / 2$ satisfying the conditions $(A)-(C)$. Since there are no indicial roots which sit in $1 / 48+\mathbb{Z}_{>0}$, the character of $V$ must be $\chi_{1,1}$ by Lemma 2. Thus it follows from Corollary 2 of Theorem 5 that $V$ is isomorphic to $L(1 / 2,0)$. However, the space spanned by characters of all simple $L(1 / 2,0)$-modules is 3 -dimensional, which contradicts to the condition (B). 
THEOREM 19. Let $V$ be a vertex operator algebra. Suppose that (A) the central charge and conformal weights are rational numbers, (B) there is a monic modular linear differential equation of order 4 whose space of solutions coincides with the space spanned by characters of all simple $V$-modules and $(\mathrm{C}) \operatorname{dim} V_{0}=1, \operatorname{dim} V_{1}=0$ and $\operatorname{dim} V_{2}=1$. Then $V$ is isomorphic to one of the minimal models $L(-46 / 3,0), L(-3 / 5,0)$, and the $\mathbb{Z}_{2}$-graded simple current extensions $\hat{L}(-114 / 7,3), \hat{L}(4 / 5,3)$ of $L(-114 / 7,0), L(4 / 5,0)$ by their simple modules with conformal weight 3 , respectively.

Proof. The conditions (A), (B) and (C) imply the rational numbers which are the candidates for central charges and indicial roots of monic MLDEs of order 4 as in Table 4. Then Propositions 14-18 prove the theorem.

As a corollary of the theorem, we obtain

COROLlary. Let $V$ be a rational and $C_{2}$-cofinite vertex operator algebra such that $V_{0}=\mathbb{C} 1, \operatorname{dim} V_{1}=0$ and $\operatorname{dim} V_{2}=1$. Suppose that there is a monic modular linear differential equation of order 4 whose space of solutions coincides with the space spanned by characters of all simple $V$-modules. Then $V$ is isomorphic to one of the minimal models with central charge $-46 / 3,-3 / 5$, and the $\mathbb{Z}_{2}$-graded simple current extensions of minimal models of central charge $-114 / 7,4 / 5$.

Proof. Since $V$ is rational and $C_{2}$-cofinite, it is known in [4, Theorem 11.3] that the central charge and conformal weights are rational numbers. Moreover, any VOA with $V_{0}=\mathbb{C} 1$ is of CFT type (see Proposition 3). Then the assertion follows from Theorem 19.

\section{Final remark.}

In this paper we classified vertex operator algebras $V$ of CFT type whose spaces of characters of simple modules coincide with the space of solutions of monic MLDEs of order 4 under the condition on the dimensions of lower homogeneous subspaces of VOAs, namely, $\operatorname{dim} V_{1}=0$ and $\operatorname{dim} V_{2}=1$. This condition provides that such VOAs are isomorphic to one of minimal models $L\left(c_{2,9}, 0\right), L\left(c_{3,5}, 0\right)$ and extensions of minimal models $L\left(c_{5,6}, 0\right), L\left(c_{3,14}, 0\right)$ by simple modules with conformal weight 3 . Therefore, this result characterizes minimal models with central charges $c_{2,9}$ and $c_{3,5}$ in terms of monic MLDEs and dimensions of $V_{1}$ and $V_{2}$. Particularly, monic MLDEs associated with data which correspond central charges and conformal weights played crucial role.

Our results (Theorems 8 and 13) can be basically used to determine a VOA whose space spanned by characters of all simple modules coincides with the space of solutions of monic MLDEs of order $n$ under the condition that the first $n-1$ coefficients of character of $V$ coincide with those of the character of $V\left(c_{p, q}, 0\right)$ when we suppose that the central charge is $c_{p, q}$. However, if we omit the condition that the central charge is $c_{p, q}$, then $\mathbb{Z}_{2^{-}}$ orbifold models of lattice VOAs and for monic MLDEs of orders 3 and 5 appear $([\mathbf{2 4}])$. The problem characterizing all VOAs associated to monic MLDEs of higher dimensional minimal models is far from at the moment. We need to develop another methods. 
The first obstruction appears for 6th order case since the space of modular form of weight 12 is 2-dimensional (The lower weight spaces have dimension 1). However, our method works if we change the condition (B) to the character of $V$ coincides with $q^{-c / 24}\left(1+q^{2}+q^{3}+2 q^{4}+2 q^{5}+m q^{6}+O\left(q^{7}\right)\right)([\mathbf{2 2}])$, which is the first 6th part of $\operatorname{ch}_{V(c, 0)}$. Moreover, combining the condition on simple modules, we state:

Conjectures. Let $V$ be a simple vertex operator algebra. Suppose that (I) the character $\operatorname{ch}_{V}$ of $V$ coincides with $q^{-c / 24} \prod_{n>1}\left(1-q^{n}\right)^{-1}$ up to the first $d$ terms with $d=(p-1)(q-1)-1$, (II) any simple $V$-module except $V$ has a character of the form $q^{h-c / 24} \prod_{1+q+O\left(q^{2}\right)}$ and (III) the space spanned by characters of all simple $V$ modules is $n=(p-1)(q-1) / 2$-dimensional and coincides with the space of a monic modular linear differential equation. Then $V$ is isomorphic to the minimal model $L\left(c_{p, q}, 0\right)$.

Unfortunately our prescription does not work on solving this conjecture since the dimension of $M_{k}(\Gamma)$ is given by $d_{k}=\sum_{i=0}^{k} \operatorname{dim} M_{2 i}(\Gamma)$ (see Table 5 ). Therefore, the general monic MLDE of order $n$ has $d_{2 n}$ linear parameters. However, while we have only $\left(d_{2 n}+2\right)$-simultaneous equations. Therefore, we cannot use our method or generalized methods to the higher order cases. For instance, by the Table 5 we can apply our method at most 9 th order case.

Table 5. Dimensions of the spaces of modular forms.

\begin{tabular}{c|c|c|c|c|c|c|c|c|c|c|c|c|c}
\hline Weight & 2 & 4 & 6 & 8 & 10 & 12 & 14 & 16 & 18 & 20 & $\cdots$ & 34 & 36 \\
\hline $\operatorname{dim} M_{k}(\Gamma)$ & 0 & 1 & 1 & 1 & 1 & 2 & 1 & 2 & 2 & 2 & $\cdots$ & 3 & 4 \\
\hline$d_{k}$ & 0 & 1 & 2 & 3 & 4 & 6 & 7 & 9 & 11 & 13 & $\cdots$ & 32 & 36 \\
\hline
\end{tabular}

Final observation is about Theorem 8 in Section 3. This theorem can be generalized even in the cases that the set of conformal weights of the simple $L\left(c_{p, q}, 0\right)$-modules has a positive integer. Therefore it seems to be very possible to classify VOAs with central charge $c_{p, q}$.

\section{Appendix by Don Zagier.}

We will show that the Diophantine equation

$$
\begin{gathered}
1050 c^{5}+(5 m+31020) c^{4}+(-703 m+275600) c^{3}+(32992 m+673104) c^{2} \\
+(-517172 m+504352) c+(3984 m-210432)=0
\end{gathered}
$$

has precisely sixteen solutions with $(c, m) \in \mathbb{Q} \times \mathbb{Z}$, seven with $m \in\{1,2\}$, four with $m>500000$, and five with $m<-100000$, as listed below in increasing order of $c$ :

$$
\begin{array}{cc|ccccccc} 
& c & -114 / 7 & -46 / 3 & -68 / 7 & -22 / 5 & -3 / 5 & 1 / 2 & 4 / 5 \\
\cline { 2 - 7 } & & 2 & 1 & 1 & 1 & 1 & 1 & 2 \\
& & c & 36 & 122 / 3 & 238 / 5 & 48 & \\
\cline { 2 - 7 } c & & & 501971 & 3132760 & 37950512 & 42987520 & \\
\hline m & & 50 & & 53 & 58 & 60 & 102 \\
\hline
\end{array}
$$


For the proof, we first notice that setting $c=x / 210$ transforms the equation into

$$
\begin{aligned}
x^{5}+ & (m+6204) x^{4}+42(-703 m+275600) x^{3}+141120(2062 m+42069) x^{2} \\
& +1852200(504352-517172 m) x+18670176000(83 m-4384)=0 .
\end{aligned}
$$

For $m \in \mathbb{Z}$ this is a monic polynomial in $x$ with integer coefficients, so that any solution with $x$ rational in fact has $x$ integral. Next, we make the substitution $m=n-x-35730$. In terms of the new variables, the equation to be solved takes on the form $A(x) n+B(x)=$ 0 , where

$$
\begin{aligned}
& A(x)=x^{4}-29526 x^{3}+290989440 x^{2}-957905978400 x+1549624608000, \\
& B(x)=420\left(1846547 x^{3}-22460023656 x^{2}+81488964629520 x-132023660227200\right)
\end{aligned}
$$

with $\operatorname{deg}(B)<\operatorname{deg}(A)$. (The coefficients in the substitution $m \mapsto n$ were chosen precisely to achieve this.) Since neither $A(x)$ nor $B(x)$ has an integral root (in fact, both are irreducible), the rational function $F(x)=B(x) / A(x)$ takes on non-zero rational values for $x \in \mathbb{Z}$, and our problem is to find all integral values in this set. Since $F$ vanishes at infinity, and all non-zero integers are at least 1 in absolute value, it is already clear that there are only finitely of these and hence only finitely many solutions $(c, m) \in \mathbb{Q} \times \mathbb{Z}$ of the original equation. A brute force calculation would be time-consuming because the set of integers $x$ for which $|F(x)| \geq 1$ is [-775532377, 775567 102], with cardinality nearly two billion. But we do not have to check the integrality of $F(x)$ for all integers $x$ in this range. Instead, we check it only for the much smaller range $|x|<50000$. This takes only 0.8 seconds with the software package PARI and yields precisely 16 values of $x$ with $F(x) \in \mathbb{Z}$, which after transforming back via $(c, m)=(x / 420,-F(x)-x-35730)$ correspond to the 16 pairs $(c, m)$ tabulated above. We then observe that for $|x|>50000$ the value of $F(x)$ belongs to the interval [-11407, 23179], and since $F(x)-n$ is irreducible for all $n$ in this interval (as PARI checks in 2.3 seconds), there are no further solutions.

\section{References}

[1] Y. Arike, M. Kaneko, K. Nagatomo and Y. Sakai, Affine vertex operator algebras and modular linear differential equations, Lett. Math. Phys., 106 (2016), 693-718.

[2] Y. Arike, K. Nagatomo and Y. Sakai, Characterization of the simple Virasoro vertex operator algebras with 2 and 3-dimensional space of characters, In: Lie algebras, vertex operator algebras, and related topics, Contemp. Math., 695, Amer. Math. Soc., Providence, 2017, 175-204.

[3] Y. Arike and K. Nagatomo, Central charges 164/5 and 236/7, preprint.

[4] C. Dong, H. Li and G. Mason, Modular-invariance of trace functions in orbifold theory and generalized Moonshine, Commun. Math. Phys., 214 (2000), 1-56.

[5] C. Dong and G. Mason, Shifted vertex operator algebras, Math. Proc. Camb. Phil. Soc., 141 (2006), 67-80.

[6] B. Feigin and D. Fuchs, Representations of the Virasoro algebra, In: Representation theory of Lie groups and related topics, Adv. Stud. Contemp. Math., 7, Gordon and Breach, New York, 1990, 465-554.

[ 7 ] B. Feigin and D. Fuchs, Verma modules over the Virasoro algebra, In: Topology (Leningrad, 1982), Lect. Notes in Math., 1984, Springer, Berlin, 1982, 230-245.

[ 8 ] I. Frenkel, Y. Huang and J. Lepowsky, On axiomatic approaches to vertex operator algebras and modules, Mem. Amer. Math. Soc., 104 (1993), no. 494.

[ 9 ] I. Frenkel and Y. Zhu, Vertex operator algebras associated to representations of affine and Virasoro 
algebras, Duke Math. J., 66 (1992), 123-168.

[10] H. R. Hampapura and S. Mukhi, Two-dimensional RCFT's without Kac-Moody symmetry, J. High Energ. Phys., 2016 (2016), No. 7, Article 138.

[11] E. L. Ince, Ordinary differential equations, Dover Publications, New York, 1944.

[12] K. Iohara and Y. Koga, Representation theory of the Virasoro algebra, Springer Monographs in Math., Springer-Verlag London, Ltd., London, 2011.

[13] M. Kaneko and M. Koike, On extremal quasimodular forms, Kyushu J. Math., 60 (2006), 457-470.

[14] C. H. Lam and H. Yamauchi, On the structure of framed vertex operator algebras and their pointwise frame stabilizers, Commun. Math. Phys., 277 (2008), 237-285.

[15] J. Lepowsky and H.-S. Li, Introduction to vertex operator algebras and their representations, Prog. Math., 227, Birkhäuser Boston, Inc., Boston, 2004.

[16] C. Marks, Irreducible vector-valued modular forms of dimension less than six, Illinois J. Math., 55 (2011), 1267-1297.

[17] G. Mason, Vector-valued modular forms and linear differential operators, Int. J. Number Theory, 3 (2007), 377-390.

[18] A. Matsuo and K. Nagatomo, Axioms for a vertex algebra and the locality of quantum fields, MSJ Memoirs, 4, Mathematical Society of Japan, 1999.

[19] S. D. Mathur, S. Mukhi and A. Sen, On the classification of rational conformal field theories, Phys. Letter B, 213 (1988), 303-308.

[20] A. Milas, Ramanujan's "Lost Notebook" and the Virasoro algebra, Commun. Math. Phys., 251 (2004), 567-588.

[21] A. Milas, Virasoro algebra, Dedekind $\eta$-function and specialized MacDonald identities, Transformation Groups, 9 (2004), 273-288.

[22] K. Nagatomo and Y. Sakai, Characterization of minimal models and 6th order modular linear differential equations, in preparation.

[23] Y. Sakai, The generalized modular linear differential equations, in preparation.

[24] M. P. Tuite and H. D. Van, On exceptional vertex operator (super) algebras, In: Developments and retrospectives in Lie theory, Dev. Math., 38, Springer, Cham, 2014, 351-385.

[25] W. Wang, Rationality of Virasoro vertex operator algebras, Internat. Math. Res. Notices, 1993 (1993), 197-211.

[26] H. Yamauchi, Module categories of simple current extensions of vertex operator algebras, J. Pure Appl. Algebra, 189 (2004), 315-328.

[27] Y. Zhu, Modular invariance of characters of vertex operator algebras, J. Amer. Math. Soc., 9 (1996), 237-302.

\section{Yusuke ARIKE}

Research Field in Education, Law, Economics and the Humanities Area

Research and Education Assembly

Kagoshima University

1-20-6, Korimoto

Kagoshima 890-0065, Japan

E-mail: arike@edu.kagoshima-u.ac.jp

\section{Yuichi SAKAI}

Multiple Zeta Research Center

Kyushu University

744, Motooka, Nishi-ku

Fukuoka 819-0395, Japan

E-mail: dynamixaxs@gmail.com

\section{Kiyokazu NaGATOMO}

Department of Pure and Applied Mathematics Graduate School of Information Science and Technology

Osaka University

Suita

Osaka 565-0871, Japan

E-mail: nagatomo@ist.osaka-u.ac.jp

\section{Don ZAGIER}

Max Planck Institute for Mathematics

Vivatsgasse 7

53111 Bonn, Germany

E-mail: dbz@mpim-bonn.mpg.de 